\title{
SOLAR NEIGHBORHOOD FLARE STARS - A REVIEW
}

\author{
W. E. KUNKEL \\ Cerro Tololo Inter-American Observatory*, La Serena, Chile
}

\section{Introduction}

Since Gershberg's (1970a) very thorough review of the subject, numerous papers dealing with the collective properties of flare stars have appeared (Petit, 1970; Kunkel, 1970d, 1973; Ambartsumian and Mirzoyan, 1971; Gurzadyan, 1971; Oskanian and Terebizh, 1971a, b; Gershberg, 1972; Veeder, 1973). The basic features of flare activity can be taken as known, then.

Physical models of flare activity have been treated by Dr Gershberg in this symposium. This review will concentrate on 'astronomical' aspects of flare activity, such as where, and under what circumstances flare activity is found in the solar vicinity. While our understanding of flare activity in clusters and associations has long enjoyed a coherent form, this cannot yet be said of the data amassed for the solar vicinity. If we hope to gain some insight into the evolution of flare activity in stars, some problems increasingly referred to in recent years must be attended to: flare activity among stars of the old disk, or even halo populations; activity on stars of spectral type earlier than that representative of the Hyades or Pleiades; and the BY Draconis syndrome, interpreted as active regions, or 'spots', on rapidly rotating stars. Duplicity among flare stars has long presented puzzling aspects that have defied elucidation, in view of strong selection effects. In addition to these topics, some ambiguities remain regarding suitable measures of flare activity, and the comparison of activities among the better known local flare stars.

Some interesting phenomena only loosely related to classical flare activity tend to be neglected in reviews and this is to be regretted. Not only is the 'non-classical' flare activity interesting in itself, but our thinking on related phenomena, such as the spots of BY Dra stars, tends to be relegated to a narrower class of stars than may be warranted by observations not properly of classical flare stars (see for example Kron, 1952; Walter, 1973).

Non-classical flare activity is briefly described (without regard for completeness) in Section 2. The influence of detection effects on flare observations is treated in Section 3. Flare stars discovered during the last four years are described in Section 4. Flare activity of local dMe stars is compared in Section 5. The BY Draconis syndrome is discussed in Section 6, followed by some remarks about rotation in Section 7. Pleiades flare activity is compared to that of the solar neighborhood in Section 8 , and evidence for the evolution of flare activity in stars is examined in Section 9, followed by some concluding remarks in Section 10.

* Operated by the Association of Universities for Research in Astronomy, Inc., under contract with the National Science Foundation. 


\section{Non Classical Flare Activity}

It will not be practical to review all forms of transient eruptive phenomena here. Many will be discussed on other occasions at this meeting. Some topics, frequently mentioned in connection with classical flare activity, have suffered long neglect, and deserve at least a superficial examination here.

Flare like activity among stars of early spectral type has been reported on six stars, listed in Table I. The earliest spectral type is B2. The heterogeneity of the group is

TABLE I

Flare stars of early spectral type

\begin{tabular}{|c|c|c|c|c|}
\hline Star & $V$ & Spectrum & Remarks & Source \\
\hline HD 037519 & 6.0 & B7 V & & Andrews (1964) \\
\hline HD 129246/7 & 3.9 ? & A2 III & Nova-like spectra (1 night) & $\begin{array}{l}\text { Ludendorff and Eberhard } \\
\text { (1905) }\end{array}$ \\
\hline HD 160202 & 6.9 & B8 e & & Bakoš (1969) \\
\hline HD 164284 & 4.8 & $\mathrm{~B} 2 \mathrm{Ve}$ & Several flares observed & Page and Page (1970) \\
\hline HD 199140 & & & BW Vul, Beta-Cephei star & Eggen (1948) \\
\hline SS 199 II & 13.0 & A0 & Halo star; RV $140 \mathrm{~km} \mathrm{~s}^{-1}$ & Philip (1968) \\
\hline
\end{tabular}

apparent. Clearly no conclusion regarding a new type of variability is warranted at this time. On the other hand, it seems less likely that we can dismiss the observations as aberrations originating in equipment, mis-identifications, or poor technique. The two emission line B stars are of particular interest. Both have been studied at some length following the initial discoveries. Two flares of HD 160202 described by Bakoš appear reminiscent of those observed on dMe stars. The slow variation, over a period of years, in quiescent light have no counterpart in dMe variability, however. The observations by Page of HD 164284 are similar to those of Bakoš: three flares brighter than $\Delta m=0.5$ were recorded in $48 \mathrm{~h}$ of monitoring. No mention is made of long-term variability in the quiescent level. Flares of these stars differ in important respects from those of $\mathrm{dMe}$ stars. They are many orders of magnitude more powerful than dMe flares. The large flare of YZ CMi observed by Andrews et al. (1969), Lovell (1969), and Kunkel (1969) produced a peak flux of about $10^{32} \mathrm{erg} \mathrm{s}^{-1}$, with a total energy output of just under $10^{35} \mathrm{erg}$. The larger event described by Bakoš (1969) appears to have reached a peak flux close to $10^{39} \mathrm{erg} \mathrm{s}^{-1}$, while lasting no longer than 20 min. While the reliability of calibration of his image orthicon equipment may be open to question, the flares reported by Page and Page (1970) for a star of earlier type were recorded photographically, where the reliability of calibration is known. The peak flux levels of these events were approximately $10^{37} \mathrm{erg} \mathrm{s}^{-1}$.

The durations of dMe flares are known to be longer on stars of higher luminosity (Haro and Chavira, 1955; Kunkel, 1967, 1973). If it were possible to apply the formula for the dMe case [Equation (15) of Kunkel (1973)], the expected durations would exceed the observed values by a factor of about ten. 
In view of the current interest in transient gamma ray phenomena (Klebesadel et al., 1973; Strong et al., 1974) it would seem encouraging to find transient events with energy levels and time scales adequate for a potential identification. The galactic distribution of the gamma-ray events is apparently isotropic, thereby excluding earlytype flare activity as a reasonable optical candidate for these events.

Among non-classical flares there are at least some that would appear to be of chromospheric origin, such as the flare observed in the calcium lines by Liller (1966) on Aldebaran.

Eruptive variability among degenerate stars is confined almost exclusively to binaries, to be discussed later at this meeting. The case of G44-32 deserves special mention. While searching for periodic variability of low amplitude, Warner et al. (1970) observed a flare with peak light $\Delta m \sim 0.6$ mag. and a duration of a few minutes in $3.5 \mathrm{~h}$ of monitoring. Since no similar occurrence is known for the possibly hundreds of hours in which white dwarfs have been observed to measure low amplitude periodic variation (Hesser et al., 1969; Hesser and Lasker, 1971) the presence of a red dwarf companion to G44-32 must be given serious consideration.

Flare activity among certain of the W Ursae Majoris stars may be related to that of dMe stars in several respects. In his monograph, Batten (1973) mentions flares observed photoelectrically on 44i Boo (Eggen, 1948), U Peg (Huruhata, 1952), and on W UMa (Kuhi, 1964). The duration of these flares are comparable to those of classical flares. From broad-band photometric colors, their spectral energy distribution would appear to be similar to that of dMe flares (Huruhata, 1952). Continuum radiation shortward of the Balmer jump seems to be involved (Kuhi, 1964). Eggen's light curves are interesting in that the flares closely resemble those observed by Huruhata, and also that an unusually deep minimum observed on one occasion might be explainable in terms of spots, which have been recognized recently to account for the photometric behavior of BY Draconis variability.

Dissimilarities should also be noted. The spectral types of W UMa stars are usually earlier than $\mathrm{K} 0$, the type normally associated with the most luminous of the classical flare stars. Also, the three flares mentioned above were all observed at an elongation.

\section{Detection Effects}

The detection of flare phenomena differs from most observing situations in that it is seldom possible to adjust the instrumental sensitivity to the signal. The effects of detection thresholds must be accounted for in detail, as a result. Oskanian and Terebizh (1971b) have discussed a procedure that attempts to account for incomplete data in which the probability of non-detection is greater than zero. Gershberg (1972) has discussed the seriousness of non-detection in a detailed comparison of observations carried out simultaneously at different stations. In Kunkel's (1973) treatment of detection, no attempt is made to compensate for incomplete detection; rather, the domain where complete detection is assured $(90 \%)$ is determined empirically, and inferences are limited to that domain. The locus of the weak event detection threshold 
is determined, and spectral optimization for the detection of flares by broad-band photometry is discussed in detail.

Problems deriving from detection effects are important not only in deciding on the presence or absence of an event in the raw data, but pervade the interpretation of a variety of statistical models of flare activity. By way of example let us consider the distribution of flare integrals $P$ defined by

$$
P=\frac{1}{I_{0}} \int_{0}^{\infty}\left(I_{f}-I_{0}\right) \mathrm{d} t
$$

where $I_{\mathrm{f}}$ and $I_{0}$ are the observed signal attributable to the flare alone and the quiescent background alone. In his study of flares observed by the working group on flare stars, Gershberg (1972) finds a distribution of flare integrals $P$ greater than a level $P_{i}$,

$$
\log _{\mathrm{e}} N\left(P>P_{i}\right)=-\beta_{G} \log _{\mathrm{e}} P_{i}+\text { const . }
$$

where the constant $\beta_{G}$ lies between 0.38 for EV Lac and 0.91 for UV Cet. The interpretation is that the major contribution to the time-averaged flare energy output is from strong events. On the other hand Kunkel (1973) finds that the conditional distribution of flare integrals for events lying within a finite range of mean durations $T_{0.5}$ (minutes) is

$$
\log _{e} N\left(P>P_{i}, \Delta T_{0.5}\right)=-\beta_{K} \log _{e} P_{i}+\text { const . }
$$

where now the coefficient $\beta_{K}$ does not depart significantly from unity. $T_{0.5}$ is the duration of a flare at half of peak light. The interpretation in this case is that strong events contribute the same, approximately, as do weaker events. The apparent difference is readily resolved by examining the detection conditions adopted in each case. The observed density distribution of flare integrals for flares of UV Ceti is shown in Figure

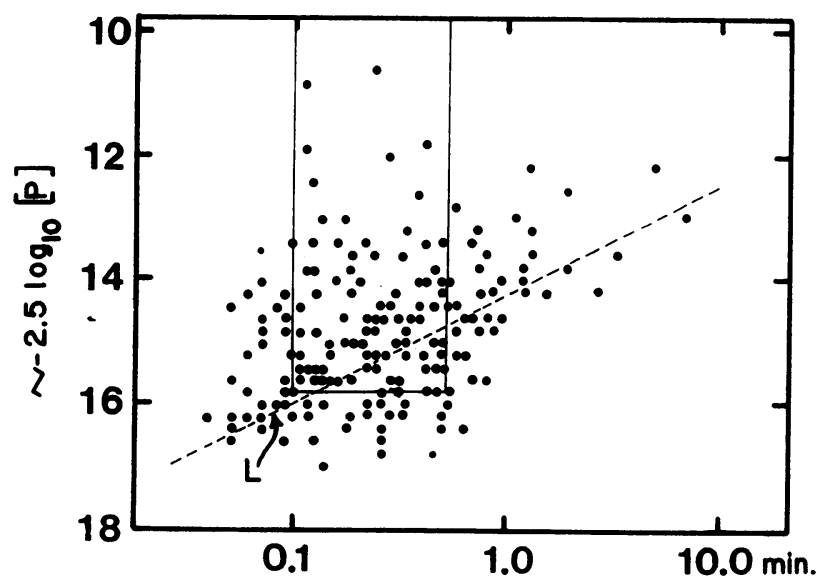

Fig. 1. Distribution of flare integrals for UV Ceti, epoch 1967.8, as a function of the logarithm of the integral (scaled as magnitudes; ordinate) and the duration at half peak light, $\boldsymbol{T}_{\mathbf{0 . 5}}$ (abcissa). See text. 
1 as a function of total energy (ordinate) and duration $T_{0.5}$ (abcissa). The detection threshold used by Gershberg considers only events brighter than $\Delta B=0 . m$. The use of a detection threshold proportional to a constant $\Delta M$ corresponds to the dashed line, since (Kunkel, 1973)

$$
-2.5 \log _{10} P \approx B-1.7 \log _{10} T_{0.5}+\text { const } .
$$

The domain examined by Kunkel is bounded by specific values of $T_{0.5}$ and a faint limit, indicated by the solid rectangle. The detection scheme of Gershberg accepts a far greater range of event durations $T_{0.5}$ for stronger flares. It is now a straightforward matter to show that both investigations describe the same distribution. For notational convenience let us put $Q=-\log _{e} P$. The distribution considered by Gershberg lies within the triangular portion formed by $\Delta B=0.3$ and the natural limit in $T_{0.5}$ at the left. $Q_{c}$ is defined as the point lying at the detection limit and natural (negative) limit in $T_{0.5}$, denoted by the letter $L$ in Figure 1 . Then we may write

$$
N(P) \propto \gamma\left(Q_{c}-Q\right) \exp \left(\beta_{K} Q\right)=\exp \left(\beta_{G} Q\right) .
$$

Taking the natural logarithm and differentiating,

$$
\mathrm{d} \log _{e} N(P)=\frac{-\mathrm{d} Q}{Q_{c}-Q}+\beta_{K} \mathrm{~d} Q=\beta_{G} \mathrm{~d} Q,
$$

so that for some level $\langle Q\rangle$ representative of the average spectral slope,

$$
\beta_{G}=\beta_{K}-\frac{1}{Q_{c}-\langle Q\rangle}
$$

The positive difference $Q_{\mathrm{c}}-\langle Q\rangle$ increases with sample size $n$. Hence

$$
\lim _{n \rightarrow \infty} \beta_{G} \rightarrow \beta_{K}
$$

The largest value of $\beta_{G}=.92$ reported by Gershberg (1972) was that for UV Cet, the largest sample studied.

It is by no means obvious which representation is to be preferred. No observational evidence is available regarding the natural limit to the distribution of flare integrals toward the right or toward the bottom of Figure 1. Since the distribution functions in current use have the undesirable property of being non-converging; that is, the integrals

$$
\int_{\infty}^{\infty} N(Q) \mathrm{d} Q-
$$

are not finite (Kunkel, 1967; Gershberg, 1970a), their application in practice requires considerable caution. A functional form such as relation (2) or (3) can be used only where its validity can be independently demonstrated from observations.

Ensemble methods such as those discussed by Gratton (1962) would appear to 
offer a better chance for resolving this matter than the unique event detection used up to now.

\section{Recently Discovered Flare Stars}

About a dozen flare stars belonging to the solar neighborhood have been discovered during the last three years. Normally only those lying within $20 \mathrm{pc}$ have been of lasting interest, the remainder being too faint to be individually important.

Although it is probably more distant, FK Serpentis represents an outstanding addition to the list of flare stars (Stienon, 1971; Hidajat, 1971; MacConnell, 1971; Herbig, 1973; Zappala, 1974). This would seem to be the best placed star for observing in some detail the flare activity of a moderately massive star (compared to masses characteristic of flare stars) still in its pre-main-sequence evolutionary state.

Among visual binaries, flare activity on the more luminous component has been discovered in at least three instances. Owen et al. (1972), using an area-scanning photometer, found that both components of EQ Pegasi are flare active; Shakhovskaya and Sofina (1972) found that the brighter component of Gliese 669 (Gliese 669b= V639 Her) is likewise active, and Kunkel (1972) found flare activity on the brighter component of Gliese 852. The fainter component was monitored for a comparable time (two hours) without noting any activity. Thus it can no longer be considered valid to assume flare activity restricted to the fainter component of a binary.

Among the newly discovered flare stars are three that have space motions characteristic of the old disk population (Kunkel, 1972). These are Gliese No.s 493.1, 852, and 866. The last of these was observed during seven flares, enough to permit an estimate of activity at a level only slightly less than that of UV Cet, a presumably young object of comparable absolute magnitude. Gliese 447 , found to flare by Lee and Hoxie (1972) is identified by Eggen (1968) as an old disk object on purely photometric grounds, as it lies $0.7 \mathrm{mag}$. below the $M_{v},(R-I)$ main sequence.

Luminous flare stars in the solar vicinity belonging to the category of 'classical' flare stars have been somewhat neglected due to their unfavorable flare visibility. These stars are important because they offer the most promising prospects for comparing the flare activity in clusters and associations with that of the solar neighborhood. YY Gem (= Gliese $278 \mathrm{C}$, spectrum dMle, $M_{v}=8.2$ for both components taken together) has long been known for unstable light, totally apart from its normal eclipses (Kron, 1952). Flare activity was found on it by Moffett and Bopp (1971). The BY Dra variable EQ Vir (=Gliese 517 , spectrum dK5e, $M_{v}=8.3$ ) was observed during a portion of a flare lasting at least two hours (Ferraz-Mello, 1973). Another star of spectral type $\mathrm{K}$ lying close to the field of $\mathrm{HZ}$ Her was observed in several low amplitude flares of short duration (Moffett and Vanden Bout, 1973). Flare activity on CC Eri was reported for the first time by Nather and Harwood (1972).

While searching for optical counterparts of X-ray sources Murdin et al. (1974) found a flare star just on the edge of the error box for 3U1636-53. The colors $(B-V=+1.45, U-B=1.2:)$ correspond to spectral type dM3. At $V=15.54$, the distance modulus is comparable to that of the Pleiades. 
None of the transient gamma-ray sources (Klebesadel et al., 1973; Strong et al., 1974) have been identified with known flare stars, or even with red dwarfs. A chromospheric origin for these events appears indicated, since the energy spectrum resembles that of solar gamma-ray events (Stecker and Frost, 1973). If the sources are galactic, red dwarfs or degenerate stars would appear to be the most promising candidates for optical counterparts, since the anisotropy of other objects would eliminate them from serious consideration.

A list of flare stars lying within $25 \mathrm{pc}$ of the Sun is given in Appendix B.

\section{Measures of Flare Activity}

In recent years numerous investigations comparing the activity of flare stars have been conducted (Kunkel, 1970d, 1973, 1974; Petit, 1970; Oskanian and Terebizh, 1971a, 1971b; Gurzadian, 1971; Gershberg and Shakhovskaya, 1971, 1973; Gershberg, 1972). The measures of activity used differ in detail, and comparison of results is not always straightforward. Regrettably the treatment of observational selection is frequently given only marginal attention.

Certain results are universally agreed upon. (1) There is a marked increase in the apparent activity with decreasing stellar luminosity. (2) The absolute energy expended through flare activity decreases with the luminosity of the star (3) About one percent or less of the total luminosity observed at visual wavelengths is attributed to flare activity.

The brief investigation of Petit (1970) elegantly establishes the first of these results.

Flare activity is commonly measured in terms of the integral under the light curve, normalized to the quiescent light preceding the onset of the flare. The resulting estimator (which has the units of time), when compared to the total observing interval, gives the fraction of the total stellar energy within a specific wavelength range radiated by flare activity. Oskanian and Terebizh (1971b), Gurzadian (1971), Gershberg and Shakhovskaya (1971), and Gershberg (1972) use this procedure. Its outstanding advantage is a result that is independent of the shape of a flare light curve. Its principal weakness is the assumption of a non-variable quiescent background, and the tendency to weigh statistical models by the measure of the integrals themselves, so that strong, infrequent flares, tend to dominate.

For reasons intrinsic to the process of detecting flares in a noisy ambient, Kunkel (1973) rejects estimates of activity based on planimetry as being somewhat prone to bias. His procedure assumes that the integral may be estimated from peak light, the duration of the event measured at half peak light, $T_{0.5}$, and a functional relation (of statistical nature) assumed to connect these with the integral. Errors as large as a factor of two in estimating the energy of individual flares are deemed acceptable in this procedure since the estimator is apparently unbiased. Errors in sampling become acceptable as the sample size increases.

A comparison of the two methods is given in Appendix A. 
The observed rate of occurrence of flares brighter than a magnitude $m$ was found in all cases to be well represented by the expression.

$$
R(m)=\exp \left[a\left(m-m_{0}\right)\right] \mathrm{h}^{-1},
$$

where $m_{0}$ is the level at which $R\left(m_{0}\right)=1$, and $a$ is a constant. The values of $a$ were found to be the same for all stars, allowing for scatter due to sampling. Thus the rate of flare activity is described solely by $m_{0}$. To facilitate comparison of levels of flare activity, a transformation of $m_{0}$ to a scale of absolute magnitudes $M_{u, 0}$ is appropriate.

The level of flare activity can be estimated for perhaps thirty stars of the solar vicinity. The quality of the estimates is uneven. Observations of more than 100 flares per star are available for only five stars. For ten stars fewer than ten flares have been reported. Solution for the constants $a$ and $m_{0}$ were carried out as follows: for samples of $\mathbf{2 0}$ or more events a least squares solution was used to estimate both constants. For smaller data sets the value of $a$ was set equal to unity, and $m_{0}$ alone was determined. A change of 0.1 in the estimate of $a$ normally results in a change in $m_{0}$ of less than 0.2 . For some stars only three events have been reported. While a 'chi-square'-distributed statistic based on so small a sample is unreliable, the uncertainty in the estimate of activity (corresponding to a standard error) is not larger than $0.8 \mathrm{mag}$.

The data are not homogeneous in other respects. About half of the reported flares were observed in the B-band. These can be scaled to the ultraviolet data on the assumption that, in the absence of photospheric 'backlight' (Kunkel, 1970a), the $(U-B)$ color of flares at peak light is $-1^{m} 1$ on the average (Kunkel, 1967, 1970a; Cristaldi and Rodonò, 1973).

Flares occurring closely spaced in time are counted as separate events by some observers and single events by others. On occasion observations of the same star taken simultaneously at different stations yield strikingly different incidence estimates; the cause would appear to be the manner in which weak events are treated, as Gershberg's (1972) investigation has shown. In this report data from different observatories at different epochs were not combined, since such a procedure does not permit testing for systematic differences between the data sets.

Estimates of flare activity are tabulated in Table II and are presented graphically in Figure 2. To achieve some measure of homogeneity preference has been given to the ultraviolet data. When only blue data were available, tabular entries in columns 5,7 , and 8 of Table II are preceded by the letter "B". For small data sets of fewer than 20 events, an assumed value for the constant $a$ is preceded by the letter ' $F$ ' in column 6 of Table II.

The activity levels of the more luminous stars are known with significantly less precision than those of the fainter stars. This is due in part to the recent growth of interest in these stars, and partly to the poor flare visibility characteristic of the more luminous flare stars. Taken together, however, the data demonstrate that strong flare activity is not uncommon in the solar vicinity to spectral type as early as dK7e.

Most of the flare activity is seen to follow an upper limit suggested by Kunkel (1970d). So few stars are shown lying well below the upper limit of activity, especially 

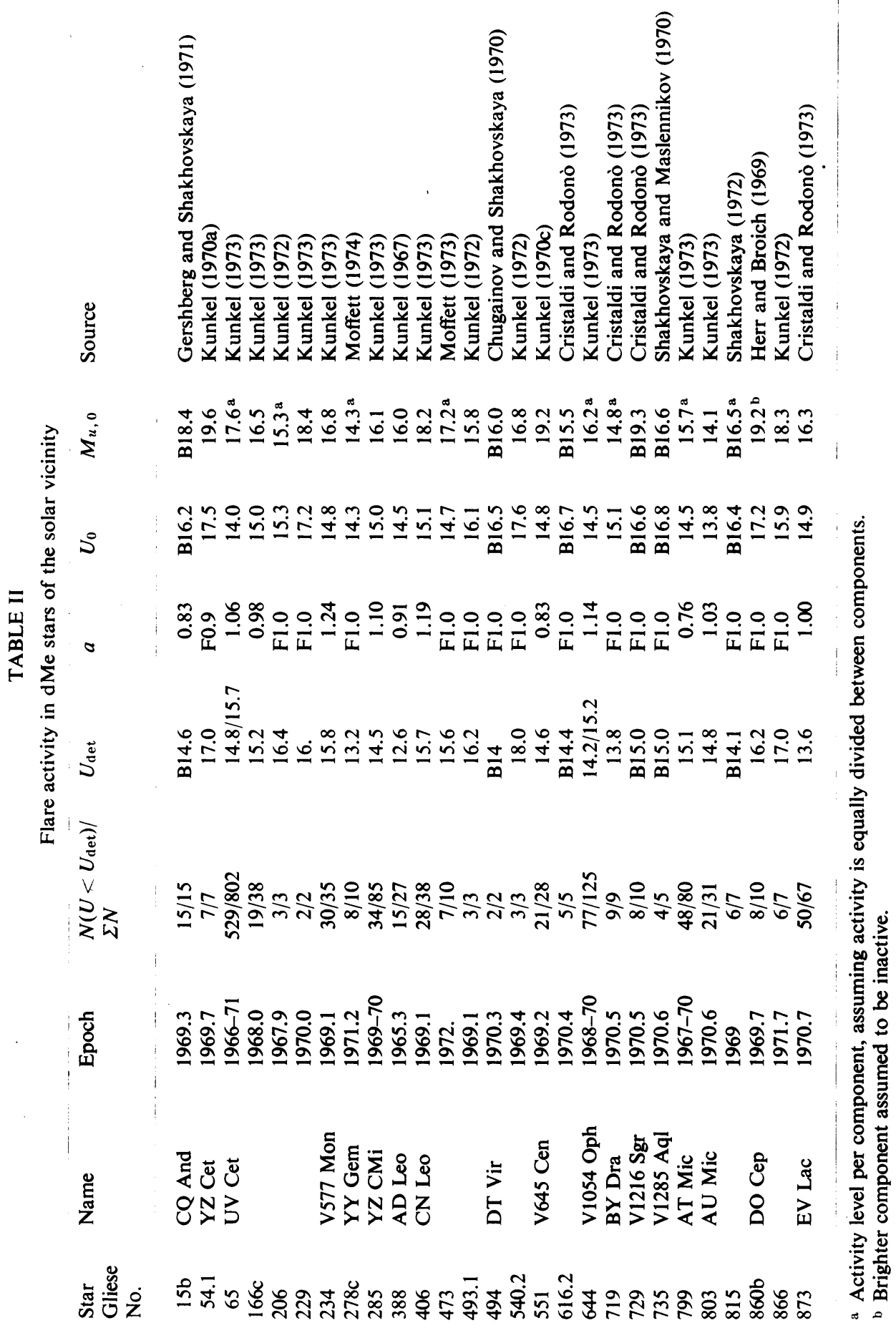


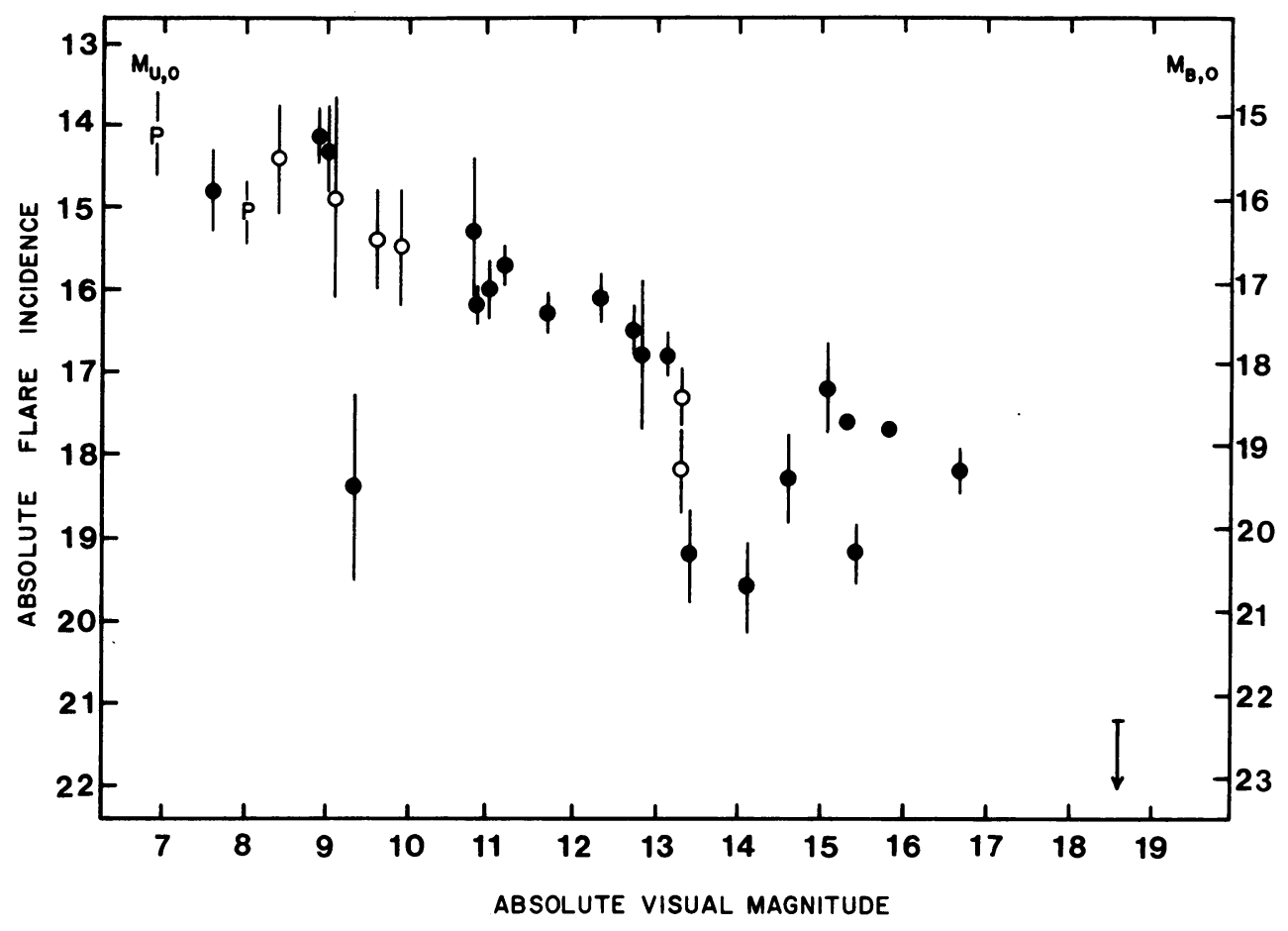

Fig. 2. Activity of solar neighborhood flare stars. Ultraviolet data (left scale) are indicated by solid symbols, and blue data (right scale) by open symbols. An upper limit ( $90 \%$ confidence) for the activity of v.B. No. 10 (Gliese $752 \mathrm{~b}$ ) is shown by the symbol at the lower right.

See Section 8 for symbols 'P'.

at greater luminosity, that the existence of objects with reduced activity is open to doubt. Information on stars at lower levels of activity is important if something is to be learned about the manner in which the flare-active phase of stellar evolution ceases. Observationally the problem is a difficult one, because one cannot easily justify long observing programs yielding only a small number of flares.

\subsection{Distributions OF AMPLITUDE AND DURATION}

dMe flares show great scatter in total energy, peak light, rise- and decay-rates, and durations. The relative frequency of peak light has been described above. The distribution of peak light follows Equation (5) over a range of at least five magnitudes (Kunkel, 1973). The distribution of total energy has been described somewhat differently by Gershberg (1972) and by Kunkel (1973). The origin of that difference is explained in Section 3.

Due to detection problems it remains unclear whether the bulk of the energy lost through flare activity is lost by a few strong events, or numerous weaker flares.

The scatter in event durations is very large: long events occasionally last more than ten times longer than short events. To facilitate an investigation of the distribution 
of flare durations, a transformation to a logarithmic scale is convenient. Putting $\left[T_{0.5}\right]=\log _{10} T_{0.5}$, Kunkel (1973) found the distribution of $\left[T_{0.5}\right]$ to be approximately Gaussian, with a standard deviation of 0.4 , irrespective of the star examined.

Kunkel (1967, 1969) and Gershberg and Chugainov (1969) found that flares of longer duration or slower rates of decay occur preferentially on the more luminous stars, confirming a result originally established by Haro and Chavira (1955). Gershberg and Shakhovskaya (1973) interpreted this as a characteristic of the flares themselves. With the somewhat larger body of data which has since then become available, Kunkel $(1973,1974)$ has presented evidence that the time-scale of flare development is virtually independent of the peak amplitude of flares, but depends instead on the stellar luminosity, in the following way:

$$
\left\langle\log _{10} T_{0.5}\right\rangle=1.084-0.119 M_{v}
$$

Kunkel has also calculated a similar relation connecting the duration of flares with the stellar surface gravity, since surface gravity may be of significance to theoretical interpretations of flare phenomena. The relation originally presented in 1969 was based on masses of but two stars, 40 Eridani C and UV Ceti. The mass of the latter has been revised twice since then. The current value (Harrington and Behall, 1973) appears to be definitive. After adding the data for V1054 Oph, a recalibration yields (Kunkel, 1973)

$$
\left\langle\log _{10} T_{0.5}\right\rangle=-(1.74 \pm 0.25) \log _{10} g \text {. }
$$

Haro and Chavira (1965) have modified their original statement, suggesting that a more complex relation is needed to describe the observations. Two effects seem important. One is purely instrumental: stronger events enjoy a more favorable signalto-noise ratio, allowing meaningful data acquisition over a greater portion of the time history of the flare. The second is that slow flares found in Orion depart significantly from the simple relations suggested by Kunkel. Haro and Chavira (1972) find that the Pleiades contain no flare stars as slow as those found in Orion. This implies that simple relations of the form suggested by Kunkel are meaningful only near the main sequence.

In his investigation of flare rise times Gershberg (1972) finds that, despite the large scatter, the more rapid rise times are those of the less luminous stars. The presence of observational selection is estimated by examining the correlation between various flare parameters. Some real correlations are found: a positive correlation between rise time and the total energy of the flare exists for all stars, and a negative correlation between the rise time and the rate of rise in light. The former could be attributed to variation in the signal-to-noise ratio. The over-all result of this investigation is clearly consistent with the findings on the durations of flares.

\subsection{Flare DistribUtion IN TIME}

The time distribution of flares has been assumed to follow Poisson incidence (Kunkel, 1967, 1970d), although evidence has been presented for certain preferred intervals 
of recurrence (Andrews, 1966; Chugainov, 1969; Osawa et al., 1968). For a time domain of a few minutes to several hours the dominance of Poisson incidence has been demonstrated by Oskanian and Terebizh (1971a). Evidence for a weak departure from Poisson incidence was found in isolated instances, in the form of a tendency toward 'clumping' of events. Precursor events suggested by Bopp and Moffett (1973) appear to be in this category. They suggest that waves originating in flares of one active region propagate over the surface of the star to another where a succeeding event is triggered, analogous to sympathetic flares of the Sun that are similarly related (Smith and Smith, 1963).

The time domain from about one hour to several days has been investigated by Chugainov (1969) and by Kunkel (1971) using autocorrelation functions. The methods differ in detail. In Chugainov's (1969) investigation of the flares of YZ CMi reported to the working group on flare stars, a rigorous autocorrelation function of the observed time series was computed. The resulting autocorrelation function may not be interpreted in terms of the autocorrelation function of a Poisson process, however. A Poisson process is a one parameter process, with the mean event rate the free parameter. The amplitude of an event does not enter other than to determine occurrence or non-occurrence of an event. The difficulty is demonstrated graphically in Figure 3.

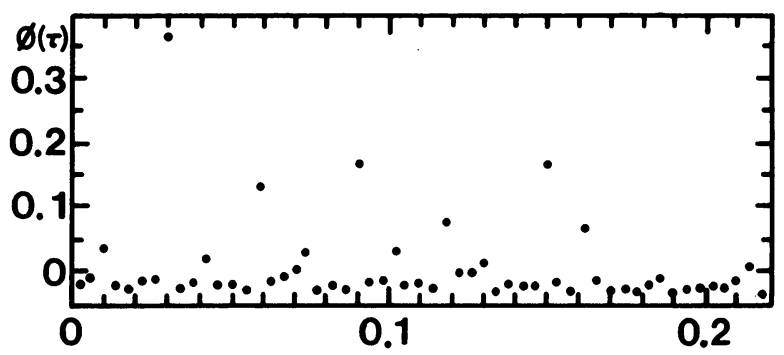

Fig. 3. Autocorrelation function of simulated flare time series prepared with random number generator. Abcissa units are fractions of the total sample duration.

Fifty flares with amplitudes distributed according to Equation (5) with $a=1$ and Poisson distributed occurrences were prepared by a random number generator. The autocorrelation function was computed following Chugainov's formula. A periodicity near $\tau=0.03$ (arbitrary time units), known to be spurious, is suggested by the figure. The occurrence of gaps in the record tends to aggravate the problem, unless weights are applied to the raw autocorrelation function which compensate for the weak sampling of time intervals most affected.

Kunkel (1971) reported finding no evidence for periodicity in the flare activity of UV Ceti, YZ CMi, and V1054 Oph. The autocorrelation function for UV Cet at epoch 1967.8 (Kunkel, 1968) is shown in Figure 4. Scaling differs from that of Chugainov. Only events brighter than $U=14^{\mathrm{m}} 2$ were counted. Estimates of the raw autocorrelation function $\phi(\tau)$ were computed by counting the number of instances in which two flares were separated by an interval between $\tau$ and $\tau+\Delta \tau$. Normalization 


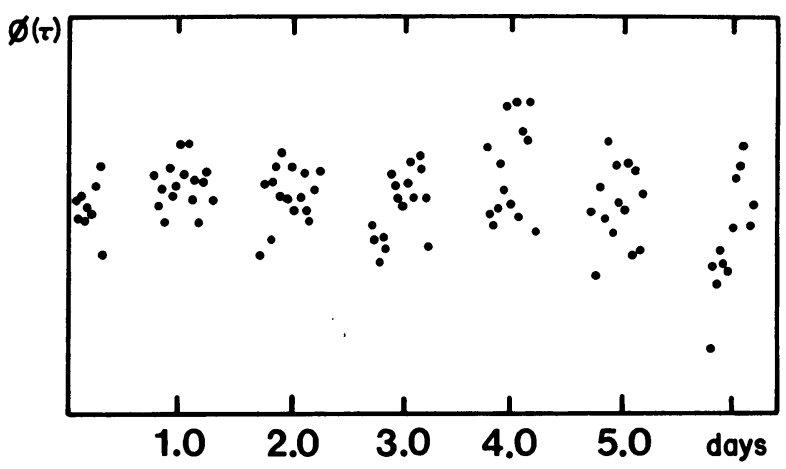

Fig. 4. Autocorrelation function of UV Ceti, epoch 1967.8 (Kunkel, 1968). Events brighter than $U=14.2$ were treated as one's, and all other points as zero's. The lag steps are $\Delta \tau=0.5 \mathrm{~h}$.

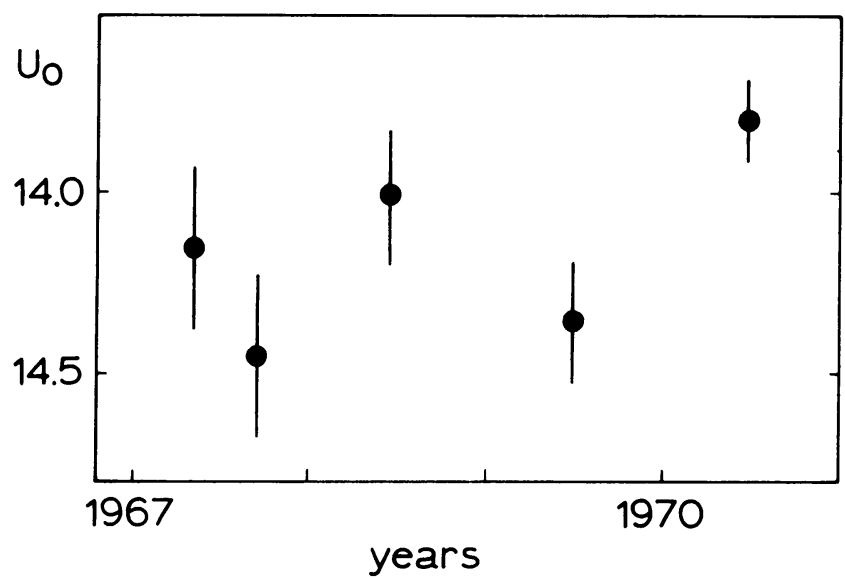

Fig. 5. Variations in the flare activity of V1054 Oph from 1967.3 to 1970.5 . Standard errors are shown.

was applied to each raw estimate using weights $w(\tau)=T(0) / T(\tau)$, where $T(\tau)$ is the length of the record for which both ends of the interval $\tau$ were observed. Autocorrelation estimates with $w(\tau)>5$ were not used. Following this algorithm a Poisson process is represented by $\phi(\tau)=$ constant (Lee, 1958).

Quantitative measures of variations in flare activity on a time scales of months to years are few. During a lapse of forty days the activity in YZ CMi was observed to change by about 40\% (Kunkel, 1971). Between 1966.8 and 1968.8 Kunkel (1973) reported a change in the level of activity of UV Ceti of also about $40 \%$. The variation in the incidence parameter $U_{0}$ for V1054 Oph from 1967.3 to 1970.5 is shown in Figure 5. Although the sampling errors are large for the earlier observations, the later observations show that some real variation is present. In all cases it should be noted that the variations are of low amplitude, so that large samples ( 50 or more events per season) are needed to continue further investigations. 


\section{The BY Draconis Syndrome}

Low amplitude light variations in red dwarfs as a phenomenon characteristic of some dMe stars were first studied on a large scale by Krzeminski and Kraft (1967) and by Krzeminski (1969). As is often the case, the earliest description of the general phenomenon predate recognition of the class phenomenon, and the significance in relation to stellar evolution and flare activity is only now emerging.

In his original examination of the photometric behavior of the eclipsing binary YY Gem, Kron (1952) proposed that the sinusoidal modulation superposed on the normal eclipse light variation is due to an uneven distribution in the surface brightness of the star. He found this variation to be temporary, with random amplitude and phase. He called attention to three other binaries showing similar photometric behavior. All of them have $\mathbf{H}$ and $\mathbf{K}$ in emission, with variation in strength. The other binaries involved stars of earlier spectral class, and an evolved component, however.

Evans' (1959) description of CC Eri presents the same phenomena described by Kron (without the classical eclipses, of course). Over a lapse of years the light variations were found to undergo a shift of phase with respect to the orbital motion of this spectroscopic binary. At the time Evans felt that the 'extraordinary duration of the eclipse phenomena' made an explanation in terms of star spots improbable.

Following a suggestion of Chugainov (1966) that a 'spot' may account for variability on BY Dra, Krzeminski and Kraft (1967) reported finding such variability only in dMe stars. In ordinary red dwarfs without Balmer line emission no variation greater than 0.02 to $0.03 \mathrm{mag}$. were found. Krzeminski (1969) again proposed non-uniform surface brightness distributions since the instances of variability in $\mathrm{dMe}$ stars in general, together with the shape of light curves and spread in periods, precludes eclipses or oscillations as likely mechanisms.

Although grey veils (Evans, 1971) appear insufficient to explain the observed phenomena (Torres and Ferraz-Mello, 1973), Bopp (1974c) finds from the radial velocity of $\mathrm{H} a$ line emission in YY Gem that occasionally some material may temporarily accumulate between the two components.

Models of spots giving surface extent and temperatures have been developed by Torres and Ferraz-Mello (1973) and by Bopp and Evans (1973). Significant surface extent is found by both groups, of up to $60^{\circ}$ and covering up to $20 \%$ of the visible hemisphere. Temperatures are estimated to be between $500^{\circ}$ and $1500^{\circ}$ cooler than the surrounding quiescent photosphere. Based on the relatively low orbital inclinations for BY Dra $\left(30^{\circ}\right)$ and CC Eri $\left(40^{\circ}\right)$, and the small observed variation in $(B-V)$, Bopp and Evans preferred the cooler spot model, thereby avoiding the need to adopt spot areas they consider unrealistically large. A grid of models covering a wide range of spot temperatures and surface extent were prepared by Torres and FerrazMello. The amplitudes and range of color variation obtained show that low inclinations pose no strong restrictions for the interpretation of actual observations. Observations of the $(V-r)$ colors point to a considerably higher spot temperature, only $500^{\circ}$ cooler than the quiescent photosphere. The $(B-V)$ color is demonstrated to be an 


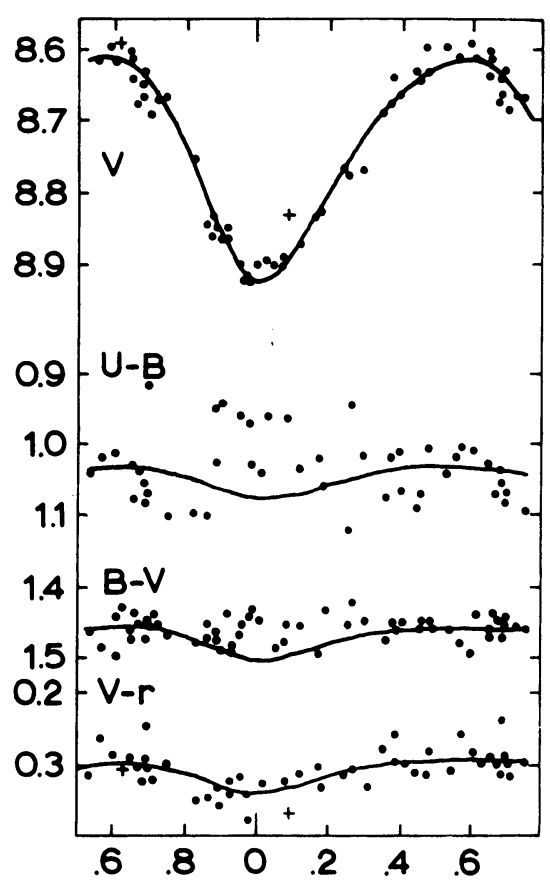

Fig. 6. Light and color variations of AU Mic, and spot model representation (Torres and Ferraz-Mello, 1973).

unreliable temperature indicator. During minimum light flare activity is strongly evident in the great scatter of $(U-B)$ (see Figure 6). Small flares with amplitudes of $\Delta U=0^{m} .1$ on a red star (for AU Mic $U-B \simeq 1^{m} \cdot 0$ and $B-V \simeq 1^{m} \cdot 4$ ) will displace the $(B-V)$ by about 0.012 mag., which is comparable to the variations expected for many conditions predicted by the model. Detectable flare activity is found on flare stars of the luminosity of AU Mic for a major fraction of the time (Kunkel, 1973), so that the contribution of flare light to the spot model is not negligible. As the effect of flare light decreases rapidly with increasing wavelength, temperatures based on the $(V-r)$ are clearly more reliable.

Chugainov (1973) has shown that photospheric variability is not a particularly permanent feature of the BY Dra syndrome. Between 1965 and 1972 the amplitude of the variation in BY Dra decreased from $\Delta B \approx 0^{m} \cdot 45$ to about $00^{m} 05$ mag., accompanied by a change in period from 3.84 to 3.79 and a decrease in $(B-V)$ from $1^{m} .24$ to $1^{m} \cdot 17$. Vogt (1973), using largely the same data, confirms these findings. The long time scale is reminiscent of the variability in the emission line spectrum of YY Gem reviewed by Kron (1952). In 35 spectra taken over a six year period (1920-1926) Sanford and Joy (1926) found noticeable variability in the line emission during this period. Twenty-five years later, Struve et al. (1950) obtained 34 spectra during an interval of 100 days in which they find virtually no variability in the strengths of the line emission. This would suggest that the susceptibility to the development of 
active regions varies with a time scale of years or decades, while the life time of the active areas themselves is one of months; individual phenomena within an active region alter their character on time scales of hours or at most days (Kron, 1952; Bopp, 1974a). Although the length of the data set for BY Dra is still too short to establish a cyclic character in the long time scale variation, an analogy to solar activity appears most inviting.

In view of the impermanent character of BY Dra variability, it would be difficult to reject the hypothesis that all flare stars are subject to such variability from time to time, as has been suggested by Bopp and Evans (1973). Some support for this view is contained in reports of variable $V, R$, or $I$ magnitudes, quite apart from ordinary flare activity, in UV Cet, CN Leo, and EV Lac (Cristaldi et al., 1969; Eggen, 1968; Chugainov et al., 1969). The better known BY Dra variables are largely spectroscopic binaries, however, and the relation between duplicity and spot phenomena is not yet understood. The nature of the observations implies that short orbital periods and nearly co-rotating stars have facilitated the detection of spots among the spectroscopic binaries.

An urgent task in future research is to assess the role of duplicity in the maintenance of flare activity. This has been anticipated by Bopp (1974b), who examined some of the better known flare stars (EV Lac, EQ Peg, AD Leo, and V1054 Oph) at a coudé dispersion of $18 \AA \mathrm{mm}^{-1}$. For these stars he is able to reject the presence of close companions in orbits with periods as short as two weeks, pointing out that close duplicity is not essential to the mechanism responsible for the maintenance of flare activity.

The existence of extensive flare activity among BY Dra variables is a recent finding. Three BY Dra variables included in Table II and Figure 2 (YY Gem, BY Dra, AU Mic) show flare activity as strong as can be found in the solar vicinity.

Robinson and Kraft (1974) have investigated the evolution of the BY Dra variability in the Pleidades and Hyades. Stars of spectral type K4 to M1 were observed in both clusters. The Pleiades sample included both flaring and non-flaring objects, while none of the Hyades stars selected are known to flare. Variability significantly larger than the observational errors was noted among three of the flaring Pleiades stars; the Hyades stars were effectively constant. We may conclude that the conditions producing BY Dra variability are closely analogous to those producing flares, and that the time scale for decay of the variability is not sensibly different from that of flare activity.

\section{Rotation}

Except for the recent discoveries on BY Draconis variables, our ignorance on matters of rotation in flare stars remains effectively as it was five years ago. Autocorrelation analyses of flare incidence remain unpersuasive. Useful upper limits on equatorial velocities have been set by Gershberg (1970b) who examined the widths of the narrow emission lines on coudé spectra of YZ CMi, AD Leo, and EV Lac. Upper limits on the projected velocity $(V \sin i)$ are 46,15 , and $25 \mathrm{~km} \mathrm{~s}^{-1}$, respectively. From their 
study of the orbital parameters of YY Gem, Struve et al. (1950) and Bopp and Evans (1973) find projected equatorial velocities less than these values.

Bopp (1974a) has examined the variation of the Balmer line emission as a function of time. The day-to-day changes observed appear to vary randomly. A time series of the equivalent width of $\mathbf{H} \beta$ for UV Ceti, sampled every $20 \mathrm{~min}$, shows a time scale for the variation on the order of hours. This is consistent with the observations of the time development of the Balmer line strengths during flares by Gershberg and Chugainov (1967) and Kunkel (1967): their results show that Balmer lines decline in a matter of hours, so that over intervals longer than this the line strengths may be expected to be uncorrelated. In this view the strength of the Balmer line emission gives a meaningful measure of activity only over such a time span, and observations spaced $24 \mathrm{~h}$ apart would be uncorrelated, and would not be a good measure of activity for the entire $24 \mathrm{~h}$ interval. It would be difficult to draw conclusions regarding rotation from data where incompleteness of sampling is this large.

Since spots persist over time scales longer than presently known rotation periods. a period-searching analysis of dMe photometry, similar to that used for studying RR Lyrae photometry (Lafler and Kinman, 1965) may prove useful in examining long photometric data sets for periods greater than a few days.

\section{Comparison with Pleiades Flare Activity}

It has been established for a long time now that flare activity in the solar neighborhood is essentially the same phenomenon as that observed by photographic means in clusters or associations (Haro, 1957; Ambartsumian, 1957; Herbig, 1962). The reported event repetition rates for these stars differ dramatically, and in a sense that would appear to run counter to the accepted view of the evolution of flare activity in stars. In Herbig's (1962) formulation, at the close of the $T$ Tauri phase of evolution, flare activity of $\mathrm{dK}$ and $\mathrm{dM}$ stars is at its highest level, declining on a time scale that is comparable to, or longer than the $\mathrm{T}$ Tauri phase. Reported flare rates in the solar vicinity are several orders of magnitude higher than those observed for association or cluster flare stars. It then becomes important to inquire if the difference is real, or if it has an explanation in terms of observing techniques: a matter of detection thresholds.

In recent years the observing programmes for the monitoring of Pleiades flare activity at Tonantzintla, Asiago, Konkoly, Bjurakan and elsewhere, have yielded a homogeneous body of data regarding flare incidence for stars of nominally unique age and composition, as well as detection thresholds. The availability of good photometry and proper motion data on the brighter of these stars make the Pleiades virtually the only sample of stars in which the analysis is straightforward.

The short investigation here described has been limited to the ultraviolet photographic material, for the sake of homogeneity. There is no assurance that the colors of flares in association and clusters, over the duration of photographic exposures, can be compared with the photoelectrically determined colors of solar neighborhood flares at peak light. Since the great majority of the ultraviolet material has been ob- 
tained at Tonantzintla (Haro, 1968; Parsamian and Chavira, 1969; Haro and Chavira, 1969, 1970, 1972; Haro and Gonzales, 1970, 1972; Haro et al., 1973) it has been used exclusively, thereby gaining confidence that the observing technique, completeness of search, and methods for estimating magnitudes, were similarly homogeneous. The data have been further restricted to only those stars that Hertzsprung (1947) included in his list as probable members of the cluster, and for which photometry (Johnson and Mitchell, 1958; Iriarte, 1973) was available. Two groups of stars stand out as suitable candidates for analysis. One is bounded by the magnitude limits $13.3<V<$ $<13.8$, covering the stars with the greatest repetition rate of events (excluding, however, star T18, which appears decidedly unusual, and T55, a Hyades member). This group is denoted as Group I; the second group is that singled out by Haro et al. (1973) as including the brightest stars with confirmed activity (Group II).

The choice of these groups is dominated by the existence of four detection modes peculiar to photographic flare observing. These are distinguished by the following: (1) whether the mean duration of a flare, in terms of $T_{0.5}$, is greater or shorter than the exposure time, and (2) whether the star is visible or not during its quiescent phase. The first of these dichotomies determines whether or not a large correction to photographic peak light estimates is necessary to achieve a comparison with photoelectric estimates (due to the integrating effects of the photographic exposure). The second determines the minimum flare light, averaged over the exposure time, required for detection: for stars visible during the quiescent state it is proportional to quiescent light (assuming a constant $\Delta m$ is required for detection), and otherwise it is a constant equal to the flux from the faintest visible star. The two groups of stars selected for examination both belong to the domain with $T_{0.5}$ less than the exposure time, and for which the quiescent state was always visible. For these the faint event detection threshold can be empirically determined from the data (Kunkel, 1973). The correction required to compare with photoelectric data can be taken from Kunkel's (1973) relation for the 'synthetic' integral, or it can be determined by numerical simulation using actual flare light curves in which the ratio of mean duration $T_{0.5}$ to exposure time is adjusted to represent the Pleiades situation.

\subsection{Group I}

Johnson and Mitchell (1958) list 19 stars with $13^{m} \cdot 3<V<13^{m} .8$. As has been pointed out by Ambartsumian et al. (1972) these stars are divided into a flaring group of nine stars, and a non-flaring group of ten stars. Since the stars are very nearly of equal brightness, their $U$-magnitudes are comparable $\left(U \approx 15^{m} 9, \pm 0.2\right)$ and they can be expected to have comparable detection thresholds. The mean flare rate for events brighter than $U_{\text {det }}=16^{\mathrm{m}} 6$ is 3.2 events per $1000 \mathrm{~h}$ in exposures averaging $12 \mathrm{~min}$ each. The probability that the non-flaring group belongs to the flaring group, but for which no events were recorded purely by chance, is less than $10^{-9}$. The flaring group is now treated as an ergodic sample: that is, time averages and ensemble averages are taken as equivalent. In the analysis the sample can be treated as a single star that has been observed for a lapse equal to nine times the actual monitoring interval. The distribu- 


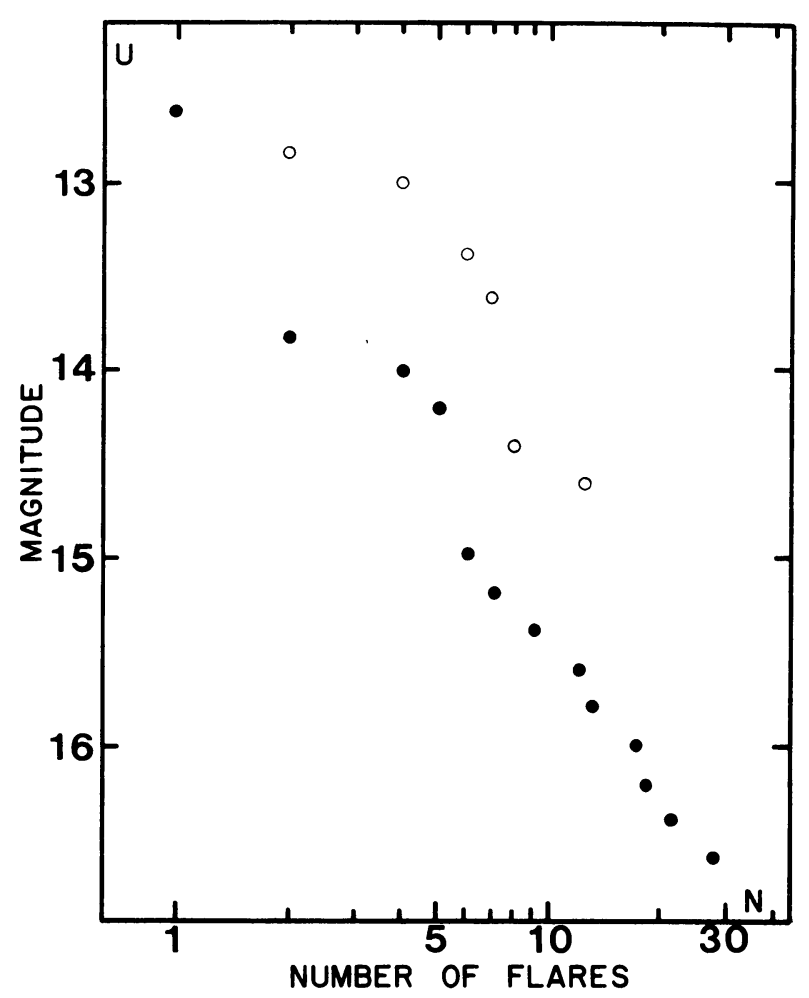

Fig. 7. Flare activity in two groups of Pleiades flare stars. Group I: solid symbols, $13.3<V<13.8$; Group II: Open symbols, $12.0<V<12.9$. Ordinate shows the number of events brighter than magnitude $U$ in an effective sampling time of about $8400 \mathrm{~h}$.

tion of flare peak light is shown in Figure 7, solid symbols. The data can be fitted by a relation giving the rate of occurrence for events brighter than level $U$ of the form $R(U)=\exp \left[a^{\prime}\left(U-U_{0}\right)\right]$. We might expect that $a^{\prime}$ will not be the same as that found in the solar neighborhood: the brightest flares in the solar neighborhood to which the relation is known to apply, (with $a=1.0$ ) have peak light of $U_{\text {abs }}>11^{m}$, on a scale of absolute magnitudes. For the Pleiades sample even at the detection threshold $U_{\text {abs }}<9.8$, where I have used a correction of $\sim 1.1$ mag. to make photographic peak light comparable to photoelectric values [equation 12, 15 of Kunkel(1973) for $M_{v} \sim 8$ ], a distance modulus of $5 m 5$, and a reddening correction to the $U$-band of $-0 m .2$ corresponding to $E(B-V)=0{ }^{m} 04$. For the solar vicinity we have no data on the distribution of flares as bright as those recorded in the Pleiades. (This is due to a total monitoring time near the Sun of less than $200 \mathrm{~h}$ for stars of comparable luminosity compared with an effective time of about $10^{4} \mathrm{~h}$ for the same star in the Pleiades). The coefficient $a^{\prime}$ was solved for by graphically weighting each flare $\Delta U$ as though it were an integral under the flare light curve (see Section 3 for the effects of detection thresholds on integrals of flare light curves) and, again by treating it as accurately representing peak light. The values obtained were 1.04 and 0.80 , respectively. The true value is taken 
to lie somewhere inbetween. The mean of the two extremes, 0.92 , lies within a standard error of the value determined for the solar neighborhood. We have then solved the incidence equation forcing the value of $a$ in Equation (5) to 0.92 (case A) and to 1.00 (case B). On a scale of absolute magnitudes the levels of activity are $M_{U, 0}=15^{m} .6$ and $15^{m} 1$ for case A and B, respectively. [See Figure 2, symbols ' $P$ ', for comparison with solar vicinity.]

\subsection{Group II}

Haro et al. (1973) list nine stars with $12^{m} \cdot 2<V<12^{m} \cdot 9\left(14^{m} \cdot 2<U<14^{m} \cdot 9\right)$ and a total of 13 flares brighter than $U_{\text {det }}=14^{\mathrm{m}} \cdot 6$. The shape of the incidence curve (Figure 7, open symbols) implies some incompleteness below $U_{\mathrm{det}}=14^{m}$, , so that only events brighter than this were used. A correction of -0.8 was used to compensate for the integrating effects of $12 \mathrm{~min}$ exposures to flares of stars at $M_{v}=7^{m}$. On a scale of absolute magnitudes the levels of activity were $M_{U, 0}=14^{\mathrm{m}} 7$ and $M_{U, 0}=14 . \mathrm{m} 1$ for case A and case $\mathrm{B}$, respectively.

Conclusions to be drawn from these data are:

(a) Although conclusive evidence is lacking (i.e., the photoelectric monitoring for flare activity), there is reason to believe that the active Pleiades dwarfs flare at the upper limit of flare activity.

(b) The reduced number of flares recorded for the more luminous group of stars is due to an inferior detection threshold, and not to reduced activity in these stars.

(c) The earliest spectral type at which strong flare activity is found in the Pleiades is that of Group II, dK3.

\section{Evolution of Flare Activity}

In the current view the flare active phase of stellar evolution is associated with the process of angular momentum loss during that portion of the pre-main sequence contraction where the star is wholly convective. For low mass dwarfs that remain largely convective on arriving at the main sequence, flare activity persists well into the main sequence phase of evolution (Haro, 1957; Ambartsumian, 1957; Herbig, 1962; Schatzman, 1962; Poveda, 1964). The rate at which angular momentum is lost is seen as correlating strongly with luminosity. Consequently the earliest spectral type within a stellar aggregate for which flare activity is found is $\mathrm{K} 0$ for ages less than $10^{7} \mathrm{yr}$ in Orion and NGC 2264, K3 at $5 \times 10^{7} \mathrm{yr}$ in the Pleiades, and M0 or M1 at $6 \times 10^{8} \mathrm{yr}$ in the Hyades. Since most flare stars of the solar neighborhood are of later spectral types, they were viewed as representing a somewhat older sample of the disk population.

In recent years the picture of the solar neighborhood flare stars has altered significantly. The earliest spectral types for flare stars are $\mathrm{K} 0$ and $\mathrm{dK} 2 \mathrm{e}$ for Butler's flare star, HD 6090 (Andrews, 1967) and PZ Mon. Also there are stars belonging to the old disk population (Kunkel, 1967, 1970d, 1972; Gershberg and Shakhovskaya, 1971 ; Lee and Hoxie, 1972; Veeder, 1974) whose activity is not significantly less than that 
of the young disk stars (Kunkel, 1970d). While flare incidence data are still lacking for the earliest spectral types, at type $\mathrm{dK} 7$ the observations are sufficiently complete to demonstrate strong activity.

These stars are expected to be younger than the more typical flare stars near the Sun. The dispersion in space motions for the ten most luminous flare stars (Gliese numbers $29.1,103,278 \mathrm{C}, 494,517,616.2,719,735,803$, and 867A) are $\sigma_{U}, \sigma_{V}$, $\sigma_{W}=24,10,11 \mathrm{~km} \mathrm{~s}^{-1}$, while for the ten least luminous flare stars (Gliese numbers $15 \mathrm{~B}, 54.1,65,406,412,473,551,752 \mathrm{~B}, 852 \mathrm{~B}$, and 866) are $\sigma_{U}, \sigma_{V}, \sigma_{W}=52,16,21 \mathrm{~km}$ $\mathrm{s}^{-1}$. Stars with the motion of the Hyades are included in both groups. Their deletion reduces the dispersion in the former group, and increases that of the latter. All are emission line objects. Following the current view, the more luminous stars would appear to be the younger group. And since the earliest spectral type within the younger grouping is earlier than that of Hyades $\mathrm{dMe}$ stars, some stars in the solar vicinity must be younger than the Hyades.

Veeder's (1973) infrared photometry of the lower main sequence confirms the relative youth of the group of luminous flare stars. He observed seven of these stars, listed in Table III.

\section{TABLE III}

Properties of luminuous late dwarf flare stars

\begin{tabular}{|c|c|c|c|c|c|c|}
\hline Star & Spectrum & $M_{\text {bol }}$ & $V-K$ & $\delta M_{\mathrm{bol}}$ & $e$ & Remarks \\
\hline $29.1^{\mathrm{a}}$ & dM0e & 7.0 & 4.10 & -1.44 & 0.067 & FF And, \\
\hline $278 \mathrm{c}^{\mathrm{b}}$ & dMle & 7.6 & 3.83 & -1.21 & 0.029 & YY Gem, \\
\hline 494 & $\mathrm{dM} 2 \mathrm{e}$ & 7.4 & 3.19 & +.23 & 0.100 & DT Vir \\
\hline 616.2 & dMle & 6.7 & 4.23 & -1.92 & 0.182 & \\
\hline $719^{a}$ & $\mathrm{dM} 0 \mathrm{e}$ & 6.2 & 3.43 & -1.31 & 0.087 & BY Dra \\
\hline 735 & $\mathrm{dM} 2 \mathrm{e}$ & 7.8 & 4.60 & -1.33 & 0.042 & $\mathrm{~V} 1285 \mathrm{Aql}$ \\
\hline $867 a^{a}$ & $\mathrm{dM} 2 \mathrm{e}$ & 7.6 & 4.33 & -1.16 & 0.003 & \\
\hline
\end{tabular}

a Spectroscopic binary

b Spectroscopic and eclipsing binary

In addition to an identifying number and spectral type, the table gives the bolometric magnitude (column 3 ), the $(V-K)$ color (column 4$)$, the displacement above the mean main sequence in the $M_{\text {bol }},(V-K)$ diagram (column 5), and the galactic orbital eccentricity (column 6 ). Of the six stars lying more than a magnitude above the main sequence, four are known spectroscopic binaries. Were one to allow for duplicity so as to minimize the displacement above the main sequence, another -0.7 mag remain to be accounted for. While the interpretation of this displacement is not yet agreed upon, its presence is definitely an indicator of relative youth. Similarly, Zappala (1973) finds that $\mathrm{dK}$ Pleiades flare stars show an infrared excess in $(V-K)$ of about 0.2 mag., which would place these stars above the main sequence in the $M_{\text {bol }},(V-K)$ diagram. A similar argument cannot be given about stars cooler than $2800 \mathrm{~K}(B-V=+1.8)$ that have been observed for flare activity. Most are found to flare, irrespective of galactic orbital parameters. The presence of flare activity on such cool stars cannot be taken as an indicator of relative youth. 


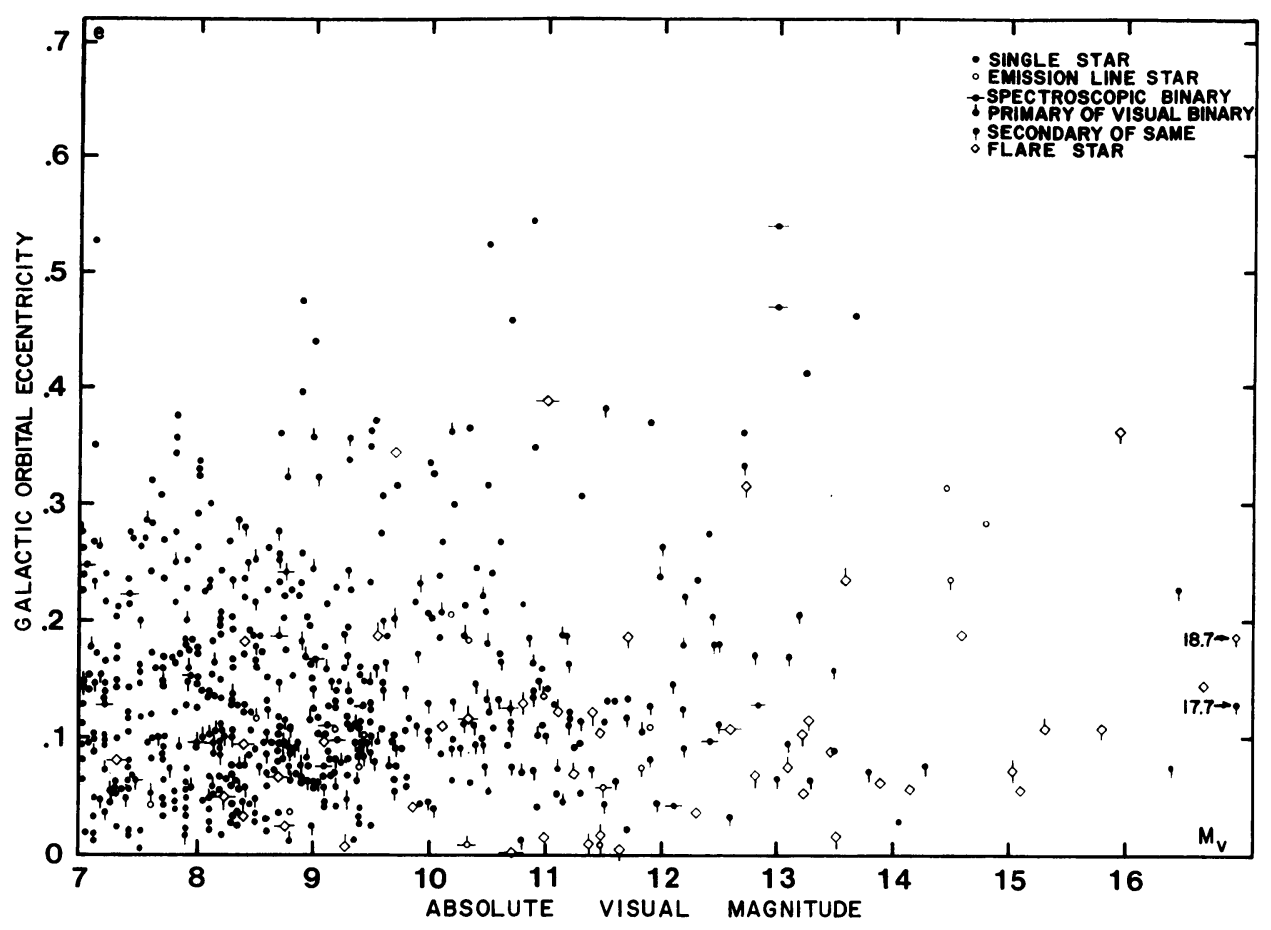

Fig. 8. Distribution of red dwarfs within $25 \mathrm{pc}$ for which trigonometric parallaxes are available (Woolley et al., 1970), as a function of galactic orbital eccentricity (ordinate) and absolute visual magnitude (abcissa).

Perhaps a more satisfactory insight into the evolution of flare activity may be gained from Figure 8. For each star with $M_{v}>7$ in the catalogue of Woolley et al. (1970) a symbol is plotted giving its position as a function of absolute magnitude and galactic orbital eccentricity. Balmer line emission is indicated by an open circle, and flare activity by an open diamond. Spectroscopic duplicity or astrometric (and visual) duplicity are indicated, respectively by a horizontal or vertical line through the symbol. The figure appears somewhat confusing at first sight. Selection effects have not been removed. Some outstanding features characteristic of flare stars are apparent at once:

(1) Flare stars in eccentric orbits are almost always components of multiple systems. SZ UMa $\left(e=0.34, M_{v}=9.8\right)$ is the notable exception; its flare activity was confirmed by Gershberg and Shakhovskaya (1971).

(2) Of the seven most luminous flare stars, five are known to be spectroscopic binaries.

(3) At the higher luminosities $\left(M_{v}<10\right)$ the proportion of flare stars among binaries is greater than among single stars.

(4) Flare and emission line stars are contained in a region bounded by an envelope extending from low eccentricities and $M_{v}=7$ to about $M_{v}=11$ at $e=0.4$.

The data are selectively incomplete at lower luminosities $\left(M_{v}>12\right)$. The abundance 
of non-flaring binaries in the range $12<M_{v}<14$ is a selection effect. These stars are often the fainter components of visual binaries favored by this display. They have not, generally, been examined for flare activity. Numerous single stars undoubtedly exist at lower luminosities, as the proper motion surveys of Giclas and Luyten demonstrate. Few of these have been put on parallax programs until recently, however.

Three conclusions may be drawn from the existence of the envelope containing flare stars. First, any point on the envelope may be considered to represent the circumstance under which the flare active phase of stellar evolution definitely ceases, even when other conditions for prolonging flare activity are the most favorable. Following the view that galactic orbital eccentricity can be taken as a rough indication of age, the flare active phase is seen to be longer lasting among the less luminous stars. Or, the more luminous the brightest flare star of a particular age group, the younger that group.

Second: It has long been known that stellar duplicity is more pronounced among $\mathrm{dMe}$ stars than among nonemission line stars. Woolley et al. give as 0.069 the fraction of spectroscopic binaries among $\mathrm{K}$ and $\mathrm{M}$ dwarfs in their catalogue. Yet among the ten most luminous flare stars five are known spectroscopic binaries, a chance occurrence of $3 \times 10^{-4}$. Equally, of the five flare stars with galactic orbital eccentricities greater than 0.2 , four are known to be binaries. From Woolley et al. (1971) we find that the fraction of binaries (spectroscopic or visual) for $\mathbf{K}$ and $\mathbf{M}$-dwarfs in this range of eccentricity is about 0.31 ; hence the probability of finding four binaries in a sample of five flare stars is less than 0.03 . Implicit in the findings, taken together with the abundance of single non-flaring stars lying below the envelope, is the generalization that stellar duplicity is significant in prolonging the duration of the flare active phase in the evolution of stars.

Third, at the lowest luminosities flare activity is found at all galactic orbital eccentricities, indicating that the duration of the flare active phase of evolution at these luminosities is comparable to, or greater than the age of the Galaxy. It would be difficult to prove, from the data presently available, that non-degenerate stars fainter than $M_{v}=15$ do not flare. Boesgaard and Hagen (1974) have determined an age for Alpha Centauri comparable to the Sun. Their result implies that the fainter component, Proxima (=V645 Cen), has the same age, consistent with the above finding.

Combining the above result with the greatest luminosity at which flare activity is found in the Hyades and Pleiades (Haro, 1968; Haro et al., 1973) one may summarize with an empirical relation for the highest luminosity of flare stars as a function of the stellar age $T$,

$$
\log _{10} T \approx 0.32 M_{v}+5.8
$$

or

$$
\log _{10} T \approx-1.2 \log _{10} L+7.0
$$

assuming an age of $5 \times 10^{7} \mathrm{yr}$ for the Pleiades and $6 \times 10^{8} \mathrm{yr}$ for the Hyades. The suggested relation holds only for luminosities of $M_{v}>6.5$. 
Flare stars belonging to moving groups are not well displayed in Figure 8. The Hyades moving group, with a galactic orbital eccentricity of about 0.1 , contributes about a third of the flare stars in the range $0.075<e<0.125$. The group associated with the Ursa Majoris stream contains no flare stars, probably because it is extremely poor in low mass stars. The possible existence of a moving group with several flare stars, and very nearly zero galactic orbital eccentricity was suggested by Kunkel (1972) on the basis of the pronounced flare activity of the stars forming the upper envelope in the diagram of flare activity (Figure 2): their space motions lie within a sphere of $10 \mathrm{~km} \mathrm{~s}^{-1}$ radius in the space motion diagram, centered on $U=-9, V=-12$, and $W=-11 \mathrm{~km} \mathrm{~s}^{-1}$. Veeder's (1973) photometry contains data for eleven stars lying within this region, as listed in Table IV; Gliese numbers 799 (=AT Mic) and 803 (=AU Mic) should have been included in the table, but were too far south to be observed by him. A significant displacement $\delta M_{\text {bol }}$ above the main sequence is apparent; being more pronounced for the more luminous spectroscopic binaries. However,

TABLE IV

Properties of stars centered on $U=-9, V=-12, W=-11 \mathrm{~km} \mathrm{~s}^{-1}$

\begin{tabular}{lllllll}
$\begin{array}{l}\text { Star } \\
(1)\end{array}$ & $\begin{array}{l}\text { Spectrum } \\
(2)\end{array}$ & $\begin{array}{l}M_{\text {bol }} \\
(3)\end{array}$ & $\begin{array}{l}V-K \\
(4)\end{array}$ & $\begin{array}{l}\delta M_{\text {bol }} \\
(5)\end{array}$ & $\begin{array}{l}e \\
(6)\end{array}$ & $\begin{array}{l}\text { Remarks } \\
(7)\end{array}$ \\
\hline 277a & dM3.5e & 8.3 & 4.65 & -.90 & 0.008 & Spectroscopic binary \\
$277 b$ & dM4.5e & 9.1 & 4.99 & -.58 & 0.008 & \\
$278 \mathrm{c}$ & dM1e & 7.6 & 3.83 & -1.21 & 0.02 & YY Gem, spectroscopic binary \\
285 & dM4.5e & 9.6 & 5.50 & -.79 & 0.037 & YZ CMi \\
382 & dM2 & 7.9 & 4.23 & -.72 & 0.032 & \\
388 & dM3.5e & 8.8 & 4.82 & -.64 & 0.017 & AD Leo double? \\
628 & dM5 & 9.8 & 5.09 & -.05 & 0.041 & Spectroscopic binary \\
$867 \mathrm{a}$ & dM2e & 7.6 & 4.33 & -1.16 & 0.003 & Spectroscopic binary \\
$867 b$ & dM3e & 9.3 & 4.98 & -.36 & 0.003 & Flares \\
876 & dM4.5 & 9.4 & 5.08 & -.40 & 0.021 & \\
$896 \mathrm{a}{ }^{2}$ & dM4e & 9.0 & 5.08 & -.80 & 0.018 & EQ Peg \\
& & & & & &
\end{tabular}

a data adjusted for contamination by fainter component.

two non-emission line single stars, Gliese 382 and 876 , also lie significantly above the main sequence. If one excludes the spectroscopic binaries, the displacement above the main sequence is $\delta M_{\text {bol }}=-0.6 \mathrm{mag}$. Veeder offers for comparison the following data regarding displacement above the main sequence for other categories: for all $\mathrm{dMe}$ stars, including flare stars, but excluding Gliese 616.2 and 735 as being too extreme, the typical displacement is -0.36 magnitudes above the main sequence. Stars showing CaII emission only, without Balmer line emission, are displaced by $-0.10 \mathrm{mag}$. If the suggested region does contain a stellar group of common origin, these stars share with the Pleiades the property that not all of the members are flare active.

The star Gliese 628 in Table IV appears anomalous. It lies very close to the main 
sequence, and if its components are at all similar, may well lie below it. The star may possibly not belong to the group. We should take note of another instance of a spectroscopic binary of low luminosity, belonging to a moving group containing a more luminous flare star: this is Wolf 629, to which Joy and Abt (1974) assigned spectral type sdM4. It is a close common proper-motion companion to the flare star V1054 Oph, Wolf 630. Both were described by Eggen (1968) as members of a moving group with an age comparable to that of M67. The spectroscopic binaries are both less luminous than $M_{v}=12$, and both appear to lie lower in the color-magnitude diagrams than other group members. Spectroscopic binaries of greater luminosity are not so affected.

\section{Concluding Remarks}

Many of the details regarding flare activity in the solar neighborhood have clarified in significant measure during the last few years, and traces of a consistent picture are emerging;

(1) flare activity of different stars is readily compared, and in the solar neighborhood stars of high activity are found at spectral types as early as $\mathrm{dK} 7 \mathrm{e}$. The level of activity is bounded by an upper limit, irrespective of other factors, such as age or duplicity.

(2) A hierarchy closely analogous to that known for associations can be recognized; the more luminous flare stars preferentially represent a younger group of stars as evidenced (1) by comparison of dispersion in space motion: it is less for the more luminous flare stars; and (2) by a displacement above the main sequence in the $M_{\text {bol }}$, $(V-K)$ diagram that is larger for the luminous flare stars than for any other group.

(3) The duration of the flare active phase of stellar evolution is a monotonically increasing function of the absolute magnitude, and is comparable to the age of the Galaxy for stars fainter than $M_{v}=15$, assuming conditions most favorable to the prolongation of the flare active phase.

(4) Duplicity appears to play an important role in prolonging the duration of the flare active phase of evolution, as shown by a diagram of galactic orbital eccentricity (an age indicator) and absolute magnitude. The incidence of duplicity is significantly higher among flare stars just inside the envelope containing flare activity than it is among $\mathbf{M}$ dwarfs in general.

(5) A comparison of the level of flare activity in the solar vicinity with that in the Pleiades shows no significant differences. The observational detection process is apparently the principal factor affecting the observed event rates.

(6) Expressed in terms of galactic dynamics, luminosity, and presence in clusters, the subset of red dwarfs affected by uneven surface brightness distribution ('spots') is not sensibly different from the set of classical flare stars. This close connection between spots and flares implies that the same physical processes are responsible for both, as in the Sun.

After summing the positive side of the ledger the negative side must be examined, since it is here that presumably most new discoveries will take place.

(1) Non-flaring dwarfs of late spectral type present a puzzle. Some of these belong 
to the young disk population, even showing infrared excesses indicative of youth. Are these single stars?

(2) Do single flare stars exist? The catalogues would seem to indicate that they do, since the majority of stars fainter than $M_{v}=15$ are listed flare or show Balmer line emission and only a fraction would be expected to be multiple. Present catalogues are strongly biased toward multiple or common proper motion stars at the lower luminosities, since the less luminous stars included tend to rely for their parallax measures on a brighter component.

(3) The relation between angular momentum and its loss through flare activity is ambiguous. If the loss of angular momentum is related to the rate of flare activity, and if that rate is restricted to some finite fraction of the luminosity, then the loss of angular momentum (or depletion of the potential for flare activity) must occur at a slower rate for the less luminous stars (Schatzman, 1962; Poveda, 1964). One may postulate a coupling of orbital angular momentum with that of individual stars to supply binaries with a larger budget of angular momentum than that available to single stars. This would account for the dominance of binaries at the envelope of the stellar ageluminosity diagram, and also for the presence of non-flaring dwarfs of comparable age and luminosity.

Exceptions not clarified by this hypothesis remain. The pair of binaries Wolf 630 and Wolf 629, which belong to the same moving group, present a situation in which the more luminous visual binary flares, while the less luminous spectroscopic binary is not known to flare, and for which no emission features have been observed spectroscopically.

(4) Cyclic activity has on occasions served as the basis for hypotheses that seek to resolve some of the above problems. At the time of writing, no dMe stars have been observed through even a single cycle of activity, although changes in the level of activity, over a time scale of years, have been reported.

\section{Appendix A}

Two methods for estimating levels of flare activity have been used. One is to form a time average of the areas under flare light curves, $\Sigma P / T$. The other is based on the observed rate of events brighter than a level, and the mean duration of flares (Kunkel, 1973).

The approximate equivalence of the two methods is shown below for those stars investigated by both techniques. The integral measures $\Sigma P_{B} / T$ were converted to a scale of bolometric magnitudes, so as to be in the same form as Kunkel's bolometric index B.I.,

$$
\text { B.I. }{ }_{B, \text { Int }}=-2.5 \log _{10}\left\{\Sigma P_{B} / T\right\}+B C_{B} .
$$

The bolometric corrections $B C_{B}$ were formed from the photometry of Iriarte (1971) and the bolometric corrections of Greenstein et al. (1970). Kunkel's (1973) bolometric index was calculated from his Equation (23). The degree of agreement is shown in 
TABLE AI

\begin{tabular}{lllll}
\multicolumn{5}{c}{ Comparison of bolometric measures of flare activity } \\
\hline Star & \multicolumn{1}{c}{$M_{v}$} & $\begin{array}{l}\text { Kunkel's } \\
\text { B.I. }\end{array}$ & B.I. $B$, Int & Difference \\
& & & & \\
AD Leo & 11.0 & 6.2 & 7.3 & -1.1 \\
EV Lac & 11.65 & 6.0 & 7.7 & -1.7 \\
YZ CMi & 12.3 & 5.9 & 7.6 & -1.8 \\
UV Cet ${ }^{a}$ & 15.5 & 5.8 & 7.3 & -1.4 \\
& & & & \\
a approximate values per component.
\end{tabular}

Table AI. A difference of about -1.5 mag. is due to corrections used in Kunkel's formula to account for flares that are either too bright or too weak to be accessible to unique event detection, and a bolometric correction (taken as the same for all flares) to account for the flare emission process (Kunkel, 1970). The scatter of 0.3 mag. (standard error) is about as narrow as can be expected in comparing activity of different epochs.

\section{Appendix B}

Data for all known flare stars believed to lie within 25 parsec* of the Sun are listed in Table BI. All stars photoelectrically observed to flare are included as well as some for which a pronounced strengthening of the Balmer emission lines was recorded on at least one occasion. The table may be considered an up-dated version of Table 1 of Gershberg and Shakhovskaya (1971).

Given in the table are the Gliese Number and a name in columns 1 and 2. The 1950 equinox coordinates appear in columns 3 and 4 . The apparent $V$ magnitude and $B-V$ color are given in columns 5 and 6 . From Veeder (1973) the $V-K$ color and bolometric absolute magnitude are given in column 7 and 9 . The absolute visual magnitude appears in column 8. A spectral type and its source is given in column 10. The source codes are those of Gliese (1969), except for the letter $J$, which refers to the catalogue of modified Mt. Wilson spectral types by Joy and Abt (1974). Parallaxes and their errors (in thousandths of a second of arc) are given in column 11. Spectroscopic parallaxes have no error estimates. The galactic orbital eccentricity given in column 11 is taken from Woolley et al. (1970). The last column contains a code denoting duplicity of those stars for which only one component appears in the table: $V=$ visual binary, $S=$ spectroscopic binary, $A=$ astrometric binary, $E=$ eclipsing binary, $C=$ member of a common proper group or pair. Both components of a binary are listed in the table if there is reason for believing that both may be flare active.

* For stars of types earlier than dK5, more distant objects are included. 
TABLE BI

\begin{tabular}{|c|c|c|c|c|c|c|c|c|c|c|c|c|c|c|c|c|c|}
\hline $\begin{array}{c}\text { GLIESE } \\
\text { NO. } \\
\end{array}$ & NAME & & R.A. & 115 & $0)^{D}$ & DECL. & v & $B-V$ & $V-K$ & $M(A B S)$ & $M(B O L)$ & SPECT. & & & $\begin{array}{l}\text { AR } \\
1-\end{array}$ & ECC. & DUP. \\
\hline $\begin{array}{l}15 . A \\
15 . B \\
29.1 \\
51 .\end{array}$ & $\begin{array}{l}\text { DM+43.44 } \\
\text { CQ AND } \\
\text { FF AND } \\
\text { HDO06090 } \\
\text { V388 CAS }\end{array}$ & $\begin{array}{l}0 \\
0 \\
0 \\
0 \\
1\end{array}$ & $\begin{array}{r}15 \\
15 \\
40 \\
58 \\
0\end{array}$ & $\begin{array}{r}31 \\
31 \\
5 \\
40 \\
8\end{array}$ & $\begin{array}{r}43 \\
43 \\
35 \\
-72 \\
62\end{array}$ & $\begin{array}{rr}3 & 44.4 \\
3 & 44.4 \\
5 & 16.4 \\
2 & 57.0 \\
2 & 5.8\end{array}$ & $\begin{array}{l}8.07 \\
11.04 \\
10.38 \\
10.58 \\
13.66\end{array}$ & $\begin{array}{l}1.56 \\
1.80 \\
1.38 \\
0.74 \\
1.68\end{array}$ & $\begin{array}{l}4.05 \\
5.06 \\
4.10 \\
5.96\end{array}$ & $\begin{array}{c}10.32 \\
13.29 \\
8.7 \\
13.89\end{array}$ & $\begin{array}{r}8.8 \\
10.9 \\
7.2 \\
10.7\end{array}$ & $\begin{array}{l}\text { OM2.5E } \\
\text { MGVE } \\
\text { DMOE } \\
\text { DKO } \\
\text { OM5E }\end{array}$ & $J$ & $\begin{array}{r}282 \\
47\end{array}$ & 07 & $\begin{array}{l}.118 \\
.067\end{array}$ & $\begin{array}{l}\mathrm{s} \\
\mathrm{s} \\
\mathrm{c}\end{array}$ \\
\hline $\begin{array}{l}54.1 \\
65.1 A \\
65.1 B \\
83.1 \\
103 .\end{array}$ & $\begin{array}{l}\text { YZ CET } \\
L 726-8 \\
\text { UV CET } \\
\text { TZ ARI } \\
\text { CC ERI }\end{array}$ & $\begin{array}{l}1 \\
1 \\
1 \\
1 \\
2\end{array}$ & $\begin{array}{r}9 \\
36 \\
36 \\
57 \\
32\end{array}$ & $\begin{array}{l}54 \\
25 \\
25 \\
28 \\
28\end{array}$ & $\begin{array}{r}-17 \\
-18 \\
-18 \\
12 \\
-44\end{array}$ & $\begin{array}{r}16.0 \\
12.7 \\
12.7 \\
50.1 \\
0.6\end{array}$ & $\begin{array}{r}12.03 \\
12.45 \\
12.95 \\
12.28 \\
8.70\end{array}$ & $\begin{array}{l}1.82 \\
1.85 \mathrm{~J} \\
1.82 \\
1.39\end{array}$ & $\begin{array}{l}6.67 \mathrm{~J} \\
5.67\end{array}$ & $\begin{array}{c}14.11 \\
15.35 \\
15.85 \\
13.91 \\
8.4\end{array}$ & $\begin{array}{l}11.7 \\
11.1\end{array}$ & $\begin{array}{l}\text { UM5.5E } \\
\text { DM5.5E } \\
\text { DM6E } \\
\text { DM6E } \\
\text { K7VE }\end{array}$ & $\begin{array}{l}J \\
J \\
J \\
E\end{array}$ & $\begin{array}{l}261 \\
381 \\
213 \\
088\end{array}$ & $\begin{array}{r}5 \\
05 \\
10 \\
12\end{array}$ & .109 & $s$ \\
\hline $\begin{array}{l}166 . \mathrm{C} \\
182 . \\
206 . \\
207.1 \\
229 .\end{array}$ & $\begin{array}{l}\text { DM- } 7.7818 \\
\text { VYS } 111 \\
\text { ROSS } 42 \\
V 371 \text { ORI } \\
\text { DM-21.1377 }\end{array}$ & $\begin{array}{l}4 \\
4 \\
5 \\
5 \\
6\end{array}$ & $\begin{array}{r}13 \\
56 \\
29 \\
31 \\
8\end{array}$ & $\begin{array}{r}4 \\
59 \\
30 \\
9 \\
28\end{array}$ & $\begin{array}{r}-7 \\
1 \\
9 \\
1 \\
-21\end{array}$ & $\begin{array}{l}44.1 \\
42.6 \\
47.3 \\
54.8 \\
50.6\end{array}$ & $\begin{array}{c}11.17 \\
9.601 \\
11.47 \\
11.68 \\
8.13\end{array}$ & $\begin{array}{l}1.68 \\
1.64 \\
1.56 \\
1.50\end{array}$ & $\begin{array}{l}4.87 \\
4.00\end{array}$ & $\begin{array}{c}12.73 \\
8.8 \\
10.73 \\
10.8 \\
9.33\end{array}$ & $\begin{array}{l}8.4 \\
7.8\end{array}$ & $\begin{array}{l}\text { DM4E } \\
\text { DMO.5E } \\
\text { OM4E } \\
\text { DM2.5E } \\
\text { OM2.5 }\end{array}$ & $\begin{array}{l}J \\
J \\
J \\
j \\
j\end{array}$ & $\begin{array}{l}205 \\
068 \\
070 \\
067 \\
174\end{array}$ & $\begin{array}{l}04 \\
08 \\
05 \\
11 \\
09\end{array}$ & $\begin{array}{l}.316 \\
.037 \\
.007 \\
.069\end{array}$ & $\begin{array}{l}v \\
s\end{array}$ \\
\hline $\begin{array}{l}234 \cdot A \\
234 \cdot B \\
277 \cdot A \\
277 \cdot B\end{array}$ & $\begin{array}{l}\text { V577 MON } \\
\text { ROSS } 614 \mathrm{~B} \\
\text { PZ MON } \\
\text { DM+36.1638A } \\
\text { ROSS } 989\end{array}$ & $\begin{array}{l}6 \\
6 \\
6 \\
7 \\
7\end{array}$ & $\begin{array}{l}26 \\
26 \\
45 \\
28 \\
28\end{array}$ & $\begin{array}{l}51 \\
51 \\
46 \\
40 \\
39\end{array}$ & $\begin{array}{r}-2 \\
-2 \\
1 \\
36 \\
36\end{array}$ & $\begin{array}{l}46.2 \\
46.2 \\
16.5 \\
19.8 \\
20.4\end{array}$ & $\begin{array}{r}11.07 \\
14.50 \\
9.50 \\
10.60 \\
11.76\end{array}$ & $\begin{array}{l}1.46 \\
1.59\end{array}$ & $\begin{array}{l}4.67 \\
4.99\end{array}$ & $\begin{array}{l}13.08 \\
16.5 \\
10.32 \\
11.48\end{array}$ & $\begin{array}{l}8.3 \\
9.1\end{array}$ & $\begin{array}{l}\text { DM4.5E } \\
\text { DK2E } \\
\text { DM3.5E } \\
\text { DM4.5E }\end{array}$ & 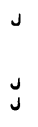 & 252 & 04 & .078 & $A$ \\
\hline $\begin{array}{l}278 . C \\
285 \\
388 \\
398 \\
406\end{array}$ & $\begin{array}{l}\text { YY GEM } \\
Y Z \text { CMI } \\
\text { AD LEO } \\
L 1113-55 \\
\text { CN LEO }\end{array}$ & $\begin{array}{r}7 \\
7 \\
10 \\
10 \\
10\end{array}$ & $\begin{array}{l}31 \\
42 \\
16 \\
33 \\
54\end{array}$ & $\begin{array}{r}26 \\
4 \\
54 \\
28 \\
6\end{array}$ & $\begin{array}{r}31 \\
3 \\
20 \\
5 \\
7\end{array}$ & $\begin{array}{r}58.8 \\
40.8 \\
7.3 \\
22.7 \\
19.2\end{array}$ & $\begin{array}{r}9.07 \\
11.23 \\
9.43 \\
12.61 \\
13.53\end{array}$ & $\begin{array}{l}1.49 \\
1.61 \\
1.54 \\
1.56 \\
2.01\end{array}$ & $\begin{array}{l}3.83 \\
5.50 \\
4.81 \\
7.46\end{array}$ & $\begin{array}{l}8.23 \\
12.34 \\
11.00 \\
11.9 \\
16.68\end{array}$ & $\begin{array}{r}7.6 \\
9.6 \\
8.8 \\
12.2\end{array}$ & $\begin{array}{l}\text { DM1E } \\
\text { OM4.5E } \\
\text { DM } 3.5 E \\
\text { DM4E } \\
\text { OM6.5E }\end{array}$ & $\begin{array}{l}J \\
J \\
J \\
J \\
J\end{array}$ & $\begin{array}{r}68 \\
167 \\
206 \\
073 \\
429\end{array}$ & $\begin{array}{l}04 \\
05 \\
04 \\
10 \\
08\end{array}$ & $\begin{array}{l}.029 \\
.037 \\
.017 \\
.110 \\
.146\end{array}$ & $\begin{array}{l}\text { SE } \\
\text { A" }\end{array}$ \\
\hline $\begin{array}{l}412 . B \\
424 . \\
4470^{\circ} \\
451.8 \\
473 . A\end{array}$ & $\begin{array}{l}\text { WX UMA } \\
\text { SZ UMA } \\
\text { ROSS } 128 \\
\text { DM+38.2285B } \\
\text { WOLF } 424\end{array}$ & $\begin{array}{l}11 \\
11 \\
11 \\
11 \\
12\end{array}$ & $\begin{array}{r}3 \\
17 \\
45 \\
50 \\
30\end{array}$ & $\begin{array}{r}2 \\
29 \\
9 \\
6 \\
51\end{array}$ & $\begin{array}{r}43 \\
66 \\
1 \\
38 \\
9\end{array}$ & $\begin{array}{r}46.7 \\
7.0 \\
6.0 \\
4.7 \\
17.6\end{array}$ & $\begin{array}{r}14.53 \\
9.32 \\
11.09 \\
12.00 \\
13.21\end{array}$ & $\begin{array}{l}1.42 \\
1.77 \\
1.82 \mathrm{~J}\end{array}$ & $\begin{array}{l}6.70 \\
3.80 \\
5.47 \\
6.43 \mathrm{~J}\end{array}$ & $\begin{array}{r}15.88 \\
9.70 \\
13.48 \\
14.98\end{array}$ & $\begin{array}{r}12.2 \\
8.4 \\
10.4 \\
11.5\end{array}$ & $\begin{array}{l}\text { OM5.5E } \\
\text { OM1.5E } \\
\text { OM4.5 } \\
\text { DM5.5E }\end{array}$ & $\begin{array}{l}J \\
J\end{array}$ & $\begin{array}{l}192 \\
119 \\
301 \\
113 \\
231\end{array}$ & $\begin{array}{l}06 \\
07 \\
05 \\
6 \\
05\end{array}$ & $\begin{array}{l}.343 \\
.090 \\
.786 \\
.073\end{array}$ & v \\
\hline $\begin{array}{l}473 . B \\
490.1 A \\
490.8 \\
493.1 \\
494.1\end{array}$ & $\begin{array}{l}\text { WOLF } 424 B \\
\text { DM+36.2322 } \\
\text { G } 164-31 \\
\text { WOLF } 461 \\
\text { DT VIR }\end{array}$ & $\begin{array}{l}12 \\
12 \\
12 \\
12 \\
12\end{array}$ & $\begin{array}{l}30 \\
55 \\
55 \\
58 \\
58\end{array}$ & $\begin{array}{r}51 \\
19 \\
18 \\
5 \\
19\end{array}$ & $\begin{array}{r}9 \\
35 \\
35 \\
5 \\
12\end{array}$ & $\begin{array}{l}17.6 \\
29.8 \\
29.6 \\
57.1 \\
38.7\end{array}$ & $\begin{array}{r}13.40 \\
10.60 \\
13.16 \\
13.34 \\
9.79\end{array}$ & $\begin{array}{l}1.42 \\
1.61 \\
1.73 \\
1.44\end{array}$ & 4.19 & $\begin{array}{r}15.2 \\
9.4 \\
11.9 \\
9.1\end{array}$ & 7.4 & $\begin{array}{l}.5 E \\
.5 E \\
E^{.5 E}\end{array}$ & $\begin{array}{l}J \\
J \\
J \\
J\end{array}$ & $\begin{array}{r}057 \\
090 \\
73\end{array}$ & 12 & $\begin{array}{l}.075 \\
.100\end{array}$ & \\
\hline $\begin{array}{l}516 . A \\
516.8 \\
517 . \\
540.2 \\
551 .\end{array}$ & $\begin{array}{l}\text { WV COM } \\
\text { G63-36B } \\
\text { DM- } 7.3646 \\
\text { ROSS } 845 \\
\text { V645 CEN }\end{array}$ & $\begin{array}{l}13 \\
13 \\
13 \\
14 \\
14\end{array}$ & $\begin{array}{l}30 \\
30 \\
32 \\
10 \\
26\end{array}$ & $\begin{array}{r}18 \\
18 \\
7 \\
26 \\
19\end{array}$ & $\begin{array}{r}17 \\
17 \\
-8 \\
-11 \\
-62\end{array}$ & $\begin{array}{r}4.2 \\
4.2 \\
5.1 \\
47.2 \\
28.1\end{array}$ & $\begin{array}{r}12.00 \\
12.30 \\
9.34 \\
13.50 \\
11.05\end{array}$ & $\begin{array}{l}1.53 \mathrm{~J} \\
1.18 \\
1.66 \\
1.97\end{array}$ & $4.64 \mathrm{~J}$ & $\begin{array}{c}11.1 \\
11.4 \\
8.3 \\
12.8 \\
15.45\end{array}$ & 11.7 & $\begin{array}{l}\text { OM3.5E } \\
\text { DM4E } \\
\text { DK5E } \\
\text { DMSE } \\
\text { DM5E }\end{array}$ & $\begin{array}{l}J \\
J \\
k \\
J\end{array}$ & $\begin{array}{r}66 \\
61 \\
072 \\
761\end{array}$ & $\begin{array}{l}13 \\
11 \\
17 \\
05\end{array}$ & $\begin{array}{r}.122 \\
.039 \\
.059\end{array}$ & $c$ \\
\hline $\begin{array}{l}569 . \\
616.2 \\
630.1 A \\
644 . A B \\
669 . A\end{array}$ & $\begin{array}{l}O M+16.2708 \\
D M+55.1823 \\
C M \text { ORA } \\
\text { V1054 OPH } \\
\text { ROSS } 868\end{array}$ & $\begin{array}{l}14 \\
16 \\
16 \\
16 \\
17\end{array}$ & $\begin{array}{l}52 \\
15 \\
33 \\
52 \\
17\end{array}$ & $\begin{array}{l}8 \\
59 \\
29 \\
48 \\
54\end{array}$ & $\begin{array}{l}16 \\
55 \\
57 \\
-8 \\
26\end{array}$ & $\begin{array}{l}18.3 \\
23.8 \\
14.8 \\
14.7 \\
32.8\end{array}$ & $\begin{array}{r}10.20 \\
9.96 \\
12.90 \\
9.76 \\
11.36\end{array}$ & $\begin{array}{l}1.48 \\
1.46 \\
1.60 \\
1.62 \mathrm{~J} \\
1.55\end{array}$ & $\begin{array}{l}4.23 \\
4.58 \mathrm{~J} \\
4.91\end{array}$ & $\begin{array}{c}10.1 \\
8.4 \\
12.0 \\
10.73 \\
11.25\end{array}$ & $\begin{array}{l}6.7 \\
8.8 \\
9.0\end{array}$ & $\begin{array}{l}\text { DM2E } \\
\text { DM1.5E } \\
\text { DM4E } \\
\text { DM3.5E } \\
\text { DM4E }\end{array}$ & $\begin{array}{l}J \\
J \\
W \\
J \\
J\end{array}$ & $\begin{array}{l}096 \\
048 \\
066 \\
156 \\
095\end{array}$ & $\begin{array}{l}10 \\
09 \\
04 \\
08\end{array}$ & $\begin{array}{r}.111 \\
.182 \\
.071\end{array}$ & $\begin{array}{l}E \\
V C\end{array}$ \\
\hline $\begin{array}{l}669.8 \\
719 . \\
729 . \\
735 .\end{array}$ & $\begin{array}{l}\text { ROSS } 867 \\
\text { FK SER } \\
\text { BY DRA } \\
\text { V1216 SGR } \\
\text { V1258 AGL }\end{array}$ & $\begin{array}{l}17 \\
18 \\
18 \\
18 \\
18\end{array}$ & $\begin{array}{l}17 \\
17 \\
32 \\
46 \\
53\end{array}$ & $\begin{array}{r}53 \\
48 \\
45 \\
45 \\
3\end{array}$ & $\begin{array}{r}26 \\
-10 \\
51 \\
-23 \\
8\end{array}$ & $\begin{array}{l}32.8 \\
13.0 \\
41.0 \\
53.5 \\
20.3\end{array}$ & $\begin{array}{c}12.92 \\
10.51 \mathrm{~J} \\
8.30 \\
10.60 \\
10.07\end{array}$ & $\begin{array}{l}1.58 \\
1.37 \mathrm{~J} \\
1.19 \\
1.75\end{array}$ & $\begin{array}{l}5.56 \\
3.96 \mathrm{~J} \\
3.46 \\
5.19 \\
4.60\end{array}$ & $\begin{array}{r}12.81 \\
7.3 \\
13.3 \\
9.9\end{array}$ & $\begin{array}{r}10.0 \\
6.2 \\
10.9 \\
7.8\end{array}$ & $\begin{array}{l}\text { DM4.5E } \\
\text { K } 4 V E \\
\text { DMOE } \\
\text { DM4.5E } \\
\text { OM3E }\end{array}$ & $\begin{array}{l}J \\
J \\
w\end{array}$ & $\begin{array}{l}064 \\
345 \\
092\end{array}$ & $\begin{array}{l}08 \\
08 \\
10\end{array}$ & $\begin{array}{l}.087 \\
.053 \\
.042\end{array}$ & $\begin{array}{l}V \\
S\end{array}$ \\
\hline $\begin{array}{l}752 . B \\
781 . \\
791.2 \\
99 . A \\
99.8\end{array}$ & $\begin{array}{l}\text { BD+04. } 40488 \\
\text { WOLF } 1130 \\
\text { HU OEL } \\
\text { AT MIC } \\
\text { AT MIC-B }\end{array}$ & $\begin{array}{l}19 \\
20 \\
20 \\
20 \\
20\end{array}$ & $\begin{array}{r}14 \\
3 \\
27 \\
38 \\
38\end{array}$ & $\begin{array}{l}32 \\
55 \\
21 \\
44 \\
44\end{array}$ & $\begin{array}{r}5 \\
54 \\
9 \\
-32 \\
-32\end{array}$ & $\begin{array}{l}4.7 \\
18.2 \\
31.2 \\
36.6 \\
36.6\end{array}$ & $\begin{array}{l}17.38 \\
12.10 \\
13.06 \\
10.92 \\
10.92\end{array}$ & $\begin{array}{l}2.12 \\
1.71 \\
1.59 \\
1.59\end{array}$ & 4.04 & $\begin{array}{l}18.57 \\
10.8 \\
13.2 \\
11.35 \\
11.35\end{array}$ & 9.4 & $\begin{array}{l}\text { OM5E } \\
\text { OM3E } \\
\text { OM6E } \\
\text { OM4.5E } \\
\text { OM } 4.5 E\end{array}$ & $\begin{array}{l}\text { J } \\
J \\
J\end{array}$ & $\begin{array}{l}173 \\
059 \\
106 \\
122 \\
122\end{array}$ & $\begin{array}{l}4 \\
05 \\
24 \\
06 \\
06\end{array}$ & $\begin{array}{r}.187 \\
.388 \\
.010\end{array}$ & $\begin{array}{l}c \\
S \\
c\end{array}$ \\
\hline : $A$ & $\begin{array}{l}\text { MUMFORD } \\
\text { AU MIC } \\
\text { G210-48A } \\
\text { G210-48B } \\
D M-51.13128\end{array}$ & $\begin{array}{l}20 \\
20 \\
20 \\
20 \\
21\end{array}$ & $\begin{array}{l}38 \\
42 \\
58 \\
58 \\
53\end{array}$ & $\begin{array}{r}54 \\
4 \\
9 \\
9 \\
35\end{array}$ & $\begin{array}{r}-69 \\
-31 \\
39 \\
39 \\
-51\end{array}$ & $\begin{array}{l}53.9 \\
31.1 \\
52.7 \\
52.7 \\
14.4\end{array}$ & $\begin{array}{r}14.68 \\
8.61 \\
10.25 \\
12.30 \\
10.40\end{array}$ & $\begin{array}{l}1.62 \\
1.44 \\
1.50 \mathrm{~J}\end{array}$ & $4.15 \mathrm{~J}$ & $\begin{array}{r}8.76 \\
9.63 \\
11.7 \\
9.6\end{array}$ & & $\begin{array}{l}\text { DM2.5E } \\
\text { OM3E } \\
M O\end{array}$ & $\begin{array}{l}J \\
J \\
H\end{array}$ & $\begin{array}{r}107 \\
75 \\
069\end{array}$ & $\begin{array}{l}06 \\
08 \\
12\end{array}$ & $\begin{array}{l}.023 \\
.188\end{array}$ & $c$ \\
\hline $\begin{array}{ll}\text { 2. } & A \\
2 . & B \\
0 . & B \\
6 . & \end{array}$ & $\begin{array}{l}\text { WOLF } 1561 \\
\text { WOLF } 1561 \mathrm{~B} \\
\text { DO CEP } \\
L 789-6 \\
\text { DM-21.6267 }\end{array}$ & $\begin{array}{l}22 \\
22 \\
22 \\
22 \\
22\end{array}$ & $\begin{array}{l}14 \\
14 \\
26 \\
35 \\
36\end{array}$ & $\begin{array}{r}42 \\
42 \\
13 \\
45 \\
1\end{array}$ & $\begin{array}{r}-9 \\
-9 \\
57 \\
-15 \\
-20\end{array}$ & $\begin{array}{r}3.0 \\
3.0 \\
26.8 \\
35.6 \\
52.8\end{array}$ & $\begin{array}{r}13.24 \\
14.50 \\
11.30 \\
12.18 \\
9.10\end{array}$ & $\begin{array}{l}1.74 \\
1.8 \\
1.96 \\
1.49\end{array}$ & 6.65 & $\begin{array}{l}13.32 \\
14.6 \\
13.3 \\
14.60 \\
9.29\end{array}$ & $\begin{array}{r}10.9 \\
7.6\end{array}$ & $\begin{array}{l}\text { DM4.5E } \\
\text { DM5E } \\
\text { DM4.5E } \\
\text { DM5.5E } \\
\text { DM2E }\end{array}$ & $\begin{array}{l}J \\
J \\
J \\
J \\
J\end{array}$ & $\begin{array}{l}104 \\
253 \\
305 \\
109\end{array}$ & $\begin{array}{l}3 \\
09 \\
11\end{array}$ & $\begin{array}{l}.237 \\
.106 \\
.188 \\
.003\end{array}$ & $\begin{array}{l}v \\
s\end{array}$ \\
\hline $\begin{array}{l}\text { 67. B } \\
71.1 A \\
71.1 B \\
73 . \\
96 . A\end{array}$ & $\begin{array}{l}L \quad 717-22 \\
L \quad 574-62 \\
L 574-61 \\
E V \quad L A C \\
D M+19.5116\end{array}$ & $\begin{array}{l}22 \\
22 \\
22 \\
22 \\
23\end{array}$ & $\begin{array}{l}36 \\
42 \\
42 \\
44 \\
29\end{array}$ & $\begin{array}{l}1 \\
18 \\
18 \\
40 \\
20\end{array}$ & $\begin{array}{r}-20 \\
-33 \\
-33 \\
44 \\
19\end{array}$ & $\begin{array}{r}52.8 \\
31.0 \\
31.0 \\
4.6 \\
39.7\end{array}$ & $\begin{array}{l}11.45 \\
13.00 P \\
14.40 P \\
10.24 \\
10.38\end{array}$ & $\begin{array}{l}1.60 \\
1.56 \mathrm{~J}\end{array}$ & 4.96 & $\begin{array}{l}11.64 \\
11.9 \\
13.3 \\
11.65 \\
11.33\end{array}$ & 9.4 & $\begin{array}{l}\text { DM3E } \\
\text { DMBE } \\
\text { DM4E } \\
\text { DM4.5E } \\
\text { DM4E }\end{array}$ & J & $\begin{array}{l}195 \\
155\end{array}$ & $\begin{array}{l}03 \\
09\end{array}$ & $\begin{array}{l}.109 \\
.018\end{array}$ & \\
\hline$B$ & EQ PEG & 23 & 29 & 20 & 19 & 39.7 & 12.40 & & & 13.4 & & UMSE & $J$ & & & & \\
\hline
\end{tabular}




\section{References for Table BI}

15. A Gershberg, R. E. and Shakhovskaya, N. I.: 1971, IAU Colloq. No. 15, Veröff. Bamberg 9, 126.

15. B Joy, A. H.: 1960, in J. L. Greenstein (ed.), Stellar Atmospheres (Vol. 6 of Stars and Stellar Systems), Univ. of Chicago Press, p. 653.

29.1 Shakhovskaya, N. I.: 1974, Info. Bull. Var. Stars, No. 897.

HD6090 Andrews, A. D.: 1966, Info. Bull. Var. Stars, No. 156.

51. Johnson, H. L. and Morgan, W. W.: 1953, Astrophys. J. 117, 323.

54.1 Kunkel, W. E.: 1970, Info. Bull. Var. Stars, No. 442.

65. AB Luyten, W. J.: 1949, Harv. Obs. Circ., No. 990.

83.1 Kunkel, W. E.: 1968, Info. Bull. Var. Stars, No. 294.

103. Nather, R. E. and Harwood, J.: 1972, IAU Circ., No. 2434.

166. C Kunkel, W. E.: 1970, Publ. Astron. Soc. Pacific 82, 1341.

182. Shakhovskaya, N. I.: 1974, Info. Bull. Var. Stars, No. 897.

206. Kunkel, W. E.: 1972, Info. Bull. Var. Stars, No. 748.

207.1 Wachmann, A. A.: 1939, Beob. Zirk. Astron. Nachr. 21, 35.

229 Kunkel, W. E.: 1973, Astrophys. J. Suppl. 25, 1.

234. AB Roques, P. E.: 1958, Publ. Astron. Soc. Pacific 70, 310.

PZ Mon Gaposhkin, S.: 1955, Bol. Tonantzintla y Tacubaya No. 13, 39.

Munch, L. and Munch, G.: 1955, Bol. Tonantzintla y Tacubaya No. 13,36.

277. B Petit, M.: 1970, Info. Bull. Var. Stars, No. 431.

278. C Moffett, T. J. and Bopp, B. W.: 1971, Astrophys. J. 168, L117.

285. van Maanen, A.: 1945, Publ. Astron. Soc. Pacific 57, 217.

388. Gordon, K. C. and Kron, G. E.: 1949, Publ. Astron. Soc. Pacific 61, 210.

398. Kunkel, W. E.: 1972, Info. Bull. Var. Stars, No. 748.

406. Sandig, K.-U.: 1951, Astron. Nachr. 280, 39.

412. B van Maanen, A.: 1949, Astrophys. J. 91, 133.

424. Gershberg, R. E. and Shakhovskaya, N. I.: 1971, IAU Colloq. No. 15, Veröff. Bamberg, 9, 216.

447. Lee, T. A. and Hoxie, D. T.: 1972, Info. Bull. Var. Stars, No. 707.

451. B van de Kamp, P.: 1969, in S. Kumar (ed.), Low Luminosity Stars, Gordon and Breach, New York, p. 129.

473. AB Kunkel, W. E.: 1967, Dissertation, Univ. of Texas, Austin.

490. B Petit, M.: 1970, Info. Bull. Var. Stars, No. 431.

493.1 Kunkel, W. E.: 1972, Info. Bull. Var. Stars, No. 748.

494. Shakhovskaya, N. I.: 1969, Info. Bull. Var. Stars, No. 361.

516. AB Roques, P. E.: 1958, Publ. Astron. Soc. Pacific 70, 310.

517. Ferraz-Mello, S.: 1973, IAU Circ. No. 2842.

540.2 Kunkel, W. E.: 1972, Info. Bull. Var. Stars, No. 748.

551. Thackeray, A. D.: 1950, Monthly Notices Roy. Astron. Soc. 110, 45.

569. Eggen, O. J.: 1967, Astrophys. J. Suppl. 16, 50.

616.2 Petit, M.: 1954, Ciel et Terre 70, 407.

630.1A Eggen, O. J. and Sandage, A. R.: 1967, Astrophys. J. 148, 911.

644. AB Eggen, O. J.: 1965, Observatory 85, 191.

669. A Shakhovskaya, N. I. and Sofina, W.: 1972, Info. Bull. Var. Soc., No. 730.

669. B Sandig, K.-U.: 1951, Astron. Nachr. 280, 39.

FK Ser Stienon, F. M.: 1971, Info. Bull. Var. Stars No. 545.

719. $\quad$ Popper, D.: 1953, Publ. Astron. Soc. Pacific 65, 278.

729. Kron, G., Gascoigne, S., and White, H. 1957, Astron. J. 62, 214.

735. Shakhovskaya, N. I. and Maslennikov, K. L.: 1970, Info. Bull. Var. Stars, No. 487.

752. B Herbig, G. H.: 1956, Publ. Astron. Soc. Pacific 68, 531.

781. Sandig, K.-U.: 1951, Astron. Nachr. 280, 39

791.2 Kunkel, W. E.: 1968, Info. Bull. Var. Stars, No. 294

799. AB Wachmann, A.: 1938, Beob. Zirk. Astron. Nachr. 21, 25.

803. Harding, G. A.: 1970, Monthly Notices Astron. Soc. South Africa 29, 130.

Mumford Mumford, G. S.: 1969, Publ. Astron. Soc. Pacific 81, 890.

815. AB Luyten, W. J.: 1925, Harv. Obs. Bull., No. 830. 
841. MacConnell, D. J.: 1968, private communication.

852. A Kunkel, W. E.: 1972, Info. Bull. Var. Stars, No. 748.

860. B Lippincott, S. L.: 1953, Publ. Astr. Soc. Pacific 65, 248.

866. Kunkel, W. E.: 1972, Info. Bull. Var. Stars, No. 748.

867. B Petit, M.: 1961, J. Observateurs 44, 11.

871.1 B Kunkel, W. E.: 1972, Info. Bull. Var. Stars, No. 748.

873. Wagman, N. E.: 1953, Harvard Ann. Card No. 1225.

896. B Roques, P. E.: 1954, Publ. Astron. Soc. Pacific 66, 256.

896. A Owen, F. N., Bopp, B. W., Moffett, T. J., and Lazor, F. J.: 1972, Astrophys. Letters $10,37$.

\section{References}

Ambartsumian, V. A.: 1957, in G. Herbig (ed.), 'Non-Stable Stars', IAU Symp. 3, 177.

Ambartsumian, V.A. and Mirzoyan, L. V.: 1971, in 'New Directions and New Frontiers in Variable Star Research', IAU Colloq. 15, Veröff. Bamberg 9, 98.

Ambartsumian, V. A., Mirzoyan, L. V., Parsamian, E. S., Chavushian, H. S., and Erastova, L. K.: 1971, Astrofizika 7, 319.

Ambartsumian, V. A., Mirzoyan, L. V., Parsamian, E. S., Chavushian, H. S., Erastova, L. K., Kazarian, E. S., and Ohanian, G. B.: 1972, Astrofizika 8, 485.

Andrews, A. D.: 1964, Irish Astron. J. 6, 212.

Andrews, A. D.: 1966, Publ. Astron. Soc. Pacific 78, 324.

Andrews, A. D.: 1967, Publ. Astron. Soc. Pacific 79, 368.

Andrews, A. D., Corvan, P., Hardy, B., Johnston, P., Johnston, W., and Perrott, J.: 1969, Info. Bull. Var. Stars No. 325.

Bakoš, G. A.: 1969, in L. Detre (ed.), Non-Periodic Phenomena in Variable Stars, Reidel, Dordrecht, p. 159.

Batten, A. H.: 1973, Binary and Multiple Systems of Stars, Pergamon Press, Oxford.

Boesgaard, A. M. and Hagen, W.: 1974, Astrophys. J. 189, 85.

Bopp, B. W.: 1974a, Monthly Notices Roy. Astron. Soc. 166, 79.

Bopp, B. W.: 1974b, Monthly Notices Roy. Astron. Soc. 168, 225.

Bopp, B. W.: 1974c, Astrophys. J. 193, 389.

Bopp, B. W. and Evans, D. S.: 1973, Monthly Notices Roy. Astron. Soc. 164, 343.

Bopp, B. W. and Moffett, T. J.: 1973, Astrophys. J. 185, 239.

Cristaldi, S. and Rodonò, M.: 1973, Astron. Astrophys. Suppl. $10,47$.

Chugainov, P. F.: 1969, in L. Detre (ed.), Non-Periodic Phenomena in Variable Stars, Reidel, Dordrecht, p. 127.

Chugainov, P. F.: 1973, Izv. Krymsk. Astrofiz. Obs. 48, 3.

Chugainov, P. F. and Shakhovskaya, N. I.: 1970, Info. Bull. Var. Stars No. 441.

Chugainov, P. F., Havlen, R. J., Westerlund, B. E., and White, R. E.: 1969, Info. Bull. Var. Stars, No. 343.

Cristaldi, S., Goddi, G., Narbone, M., and Rodonò, M.: 1969, in L. Detre (ed.), Non-Periodic Phenomena in Variable Stars, Reidel, Dordrecht, p. 149.

Eggen, O. J.: 1948, Astron. J. 53, 197 (Abstract).

Eggen, O. J.: 1968, Astrophys. J. Suppl. 16, 49.

Eggen, O. J.: 1971, Astrophys. J. Suppl. 22, 389.

Evans, D. S.: 1959, Monthly Notices Roy. Astron. Soc. 119, 526.

Evans, D. S.: 1971, Monthly Notices Roy. Astron. Soc. 154, 329.

Ferraz-Mello, S.: 1973, IAU Circ. No. 2842.

Gershberg, R. E.: 1970a, Flares of Red Dwarf Stars, English translation by D. J. Mullan, Armagh.

Gershberg, R. E.: 1970b, in A. Slettebak (ed.), Stellar Rotation, Reidel, Dordrecht, p. 249.

Gershberg, R. E.: 1972, Astrophys. Space Sci. 19, 75.

Gershberg, R. E. and Chugainov, P. F.: 1967, Astron. Zh. 44, 260.

Gershberg, R. E. and Chugainov, P. F.: 1969, Izv. Krymsk. Astrofiz. Obs. $40,7$.

Gershberg, R. E. and Shakhovskaya, N. I.: 1971, in 'New Directions and New Frontiers in Variable Star Research', IAU Colloq. 15, Veröff. Bamberg 9, 126.

Gershberg, R. E. and Shakhovskaya, N. I.: 1973, Nature, Phys. Sci. 242, 85. 
Gliese, W.: 1969, Veröff. Astron. Rechen Inst. Heidelberg No. 22.

Gratton, L.: 1962, Soc. Astron. Italiana, Atti del Convegno Astron. di Milano-Merate.

Greenstein, J. L., Neugebauer, G., and Becklin, E. E.: 1970, Astrophys. J. 161, 519.

Gurzadian, G. A.: 1971, Bol. Tonantzintla Tacubaya 6, 39.

Haro, G.: 1957, in G. Herbig (ed.), 'Non-Stable Stars', IAU Symp. 3, 96.

Haro, G.: 1968, in B. M. Middlehurst and L. H. Aller (eds.), Nebulae and Interstellar Matter (Stars and Stellar Systems 7), 141, University of Chicago Press, Chicago.

Haro, G. and Chavira, E.: 1955, Bol. Tonantzintla Tacubaya No. 12.

Haro, G. and Chavira, E.: 1965, Vistas Astron. 8, 89.

Haro, G. and Chavira, E.: 1969, Bol. Tonantzintla Tacubaya 5, 23.

Haro, G. and Chavira, E.: 1970, Bol. Tonantzintla Tacubaya 5, 181.

Haro, G. and Chavira, E.: 1972, Bol. Tonantzintla Tacubaya 6, 155.

Haro, G. and Gonzales, G.: 1970, Bol. Tonantzintla Tacubaya 5, 191.

Haro, G. and Gonzales, G.: 1972, Bol. Tonantzintla Tacubaya 6, 149.

Haro, G., Chavira, E., and Gonzales, G.: 1973, Bol. Inst. Tonantzintla 1, 3.

Harrington, R. S. and Behall, A. L.: 1973, Astron. J. 78, 1096.

Herbig, G.: 1962, in J. Sahade (ed.), Symposium on Stellar Evolution, Astron. Obs. Nat. Univ. La

Plata, La Plata, p. 45.

Herbig, G.: 1973, Astrophys. J. 182, 129.

Herr, R. B. and Broich, J. A.: 1969, Info. Bull. Var. Stars No. 329.

Hertzsprung, E.: 1947, Bull. Astron. Inst. Neth. No. 11.

Hesser, J. E. and Lasker, B. M.: 1971, in 'New Directions and New Frontiers in Variable Star' Research', IAU Colloq. 15, Veröff. Bamberg 9, 98.

Hesser, J. E., Ostriker, J. P., and Lawrence, G. M.: 1969 Astrophys. J. 155, 919.

Hidajat, B.: 1971, Info. Bull. Var. Stars No. 559.

Huruhata, M.: 1952, Publ. Astron. Soc. Pacific 64, 200.

Iriarte, B.: 1971, Bol. Tonantzintla Tacubaya 6, 143.

Iriarte, B.: 1973, Info. Bull. Var. Stars No. 875.

Johnson, H. L. and Mitchell, R. I.: 1958, Astrophys. J. 128, 31.

Joy, A. H. and Abt, H. A.: 1974, Astrophys. J. Suppl. 28, 1.

Klebesadel, R. W., Strong, I. B., and Olson, R. A.: 1973, Astrophys. J. 182, L85.

Kraft, R. P. and Greenstein, J. L.: 1969, in S. Kumar (ed.), Low Luminosity Stars, Gordon and Breach, New York, p. 65.

Kron, G. E.: 1952, Astrophys. J. 115, 301.

Krzeminski, W.: 1969, in S. Kumar (ed.), Low Luminosity Stars, Gordon and Breach, New York, p. 57.

Krzeminski, W. and Kraft, R. P.: 1967, Astron. J. 72, 307.

Kuhi, L. V.: 1964, Publ. Astron. Soc. Pacific 76, 430.

Kunkel, W. E.: 1967, Dissertation, University of Texas, Austin.

Kunkel, W. E.: 1968, Info. Bull. Var. Stars No. 315.

Kunkel, W. E.: 1969, Nature 222, 1129.

Kunkel, W. E.: 1970a, Astrophys. J. 161, 503.

Kunkel, W. E.: 1970b, Info. Bull. Var. Stars No. 442.

Kunkel, W. E.: 1970c, Info. Bull. Var. Stars No. 462.

Kunkel, W. E.: 1970d, Publ. Astron. Soc. Pacific 82, 1341.

Kunkel, W. E.: 1971, Bull. Am. Astron. Soc. 3, 13.

Kunkel, W. E.: 1972, Info. Bull. Var. Stars No. 748.

Kunkel, W. E.: 1973, Astrophys. J. Suppl. 25, 1.

Kunkel, W. E.: 1974, Nature 248, 571.

Lafler, J. and Kinman, T. D.: 1965, Astrophys. J. Suppl. 11, 216.

Lee, Y. W.: 1958, Statistical Theory of Communication, Wiley, New York.

Lee, T. A. and Hoxie, D. T.: 1972, Info. Bull. Var. Stars No. 707.

Liller, W.: 1966, Astron. J. 71, 862.

Lovell, B.: 1969, Nature 222, 1126.

Ludendorff, H. and Eberhard, G.: 1905, Astron. Nachr. 170, 165.

MacConnell, D. J.: 1968, Astrophys. J. 153, 313.

MacConnell, D. J.: 1971, Info. Bull. Var. Stars No. 570. 
Moffett, T. J.: 1973, Monthly Notices Roy. Astron. Soc. 164, 11.

Moffett, T. J.: 1974, private communication.

Moffett, T. J. and Bopp, B. W.: 1971, Astrophys. J. 168, L117.

Moffett, T. J. and Vanden Bout, P. A.: 1973, Info. Bull. Var. Stars, No. 833.

Murdin, P. G., Penston, M. J., Penston, M. V., Glass, I. S., Sanford, P. W., Hawkins, F., Mason, K., and Willmore, A. P.: 1974, Monthly Notices Roy. Astron. Soc. 169, 23.

Nather, R. E. and Harwood, J.: 1972, IAU Circ. No. 2434.

Osawa, K., Ichimura, K., Noguchi, T., and Watanabe, E.: 1968, Tokio Astron. Bull., 2nd Series, No. 180.

Oskanian, V. S. and Terebizh, V. Yu.: 1971a, Astrofizika 7, 1.

Oskanian, V. S. and Terebizh, V. Yu.: 1971b, Astrofizika 7, 281.

Owen, F. N., Bopp, B. W., Moffett, T. J., and Lazor, F. J.: 1972, Astrophys. J. 10, 37.

Parsamian, E. and Chavira, E.: 1969, Bol. Tonantzintla Tacubaya 5, 35.

Page, A. A. and Page, B.: 1970, Proc. Astron. Soc. Australia 1, 324.

Petit, M.: 1970, Info. Bull. Var. Stars, No. 430.

Philip, A. G. D.: 1968, Publ. Astron. Soc. Pacific 80, 171.

Poveda, A.: 1964, Nature 202, 1319.

Robinson, E. L. and Kraft, R. P.: 1974, Astron. J. 79, 698.

Sanford, R. F. and Joy, A. H.: 1926, Astrophys. J. 64, 250.

Shakhovskaya, N. I.: 1972, Izv. Krim. Astrophys. Obs. 45, 124.

Shakhovskaya, N. I. and Maslennikov, K. L.: 1970, Info. Bull. Var. Stars, No. 487.

Shakhovskaya, N. I. and Sofina, W.: 1972, Info. Bull. Var. Stars, No. 730.

Schatzman, E.: 1962, Ann. Astrophys. 25, 18.

Smith, H. J. and Smith, E. V. P.: 1963, Solar Flares, MacMillan, New York, p. $92 \mathrm{ff}$.

Stecker, F. W. and Frost, K. J.: 1973, Nature 245, 70.

Stienon, F. M.: 1971, Info. Bull. Var. Stars No. 545.

Strong, I. B., Klebesadel, R. W. and Olson, R. A.: 1974, Astrophys. J. 188, L1.

Struve, O., Herbig, G. H. and Horak, H.: 1950, Astrophys. J. 112, 216.

Torres, C. A. O. and Ferraz-Mello, S.: 1973, Astron. Astrophys. 27, 231.

Veeder, G. J.: 1973, Dissertation, Cal. Tech., Pasadena.

Veeder, G. J.: 1974, Astron. J. 79, 702.

Vogt, S. S.: 1973, Bull. Am. Astron. Soc. 5, 399.

Walter, K.: 1973, Astrophys. Space Sci. Rev. 21, 289.

Warner, B., van Citters, G. W., and Nather, R. G.: 1970, Nature 226, 67.

Wilson, O. C. and Woolley, R.: 1970, Monthly Notices Roy. Astron. Soc. 148, 463.

Woolley, R., Epps, E. A., Penston, M. J., and Pocock, S. B.: 1970, Roy. Obs. Ann. No. 5.

Woolley, R., Pocock, S. B., Epps, E. A., and Flim, R.: 1971, Roy. Obs. Bull. No. 166.

Zappala, R. R.: 1973, Annual Report of the Director, Hale Observatories.

Zappala, R. R.: 1974, Astrophys. J. 187, 257. 